\title{
Ammonia in infrared dark clouds
}

\author{
T. Pillai ${ }^{1}$, F. Wyrowski ${ }^{1}$, S. J. Carey ${ }^{2}$, and K. M. Menten ${ }^{1}$
}

\author{
1 Max-Planck-Institut für Radioastronomie, Auf dem Hügel 69, 53121 Bonn, Germany \\ e-mail: [thushara; wyrowski ; menten] @mpi fr-bonn .mpg .de \\ 2 Spitzer Science Center, California Institute of Technology, MC 314-6, 1200 East California Boulevard, Pasadena, CA 91125, USA \\ e-mail: carey@ipac.caltech.edu
}

Received 30 August 2005 / Accepted 5 December 2005

\section{ABSTRACT}

Context. While low mass clouds have been relatively well studied, our picture of high-mass star formation remains unclear. Infrared Dark Clouds appear to be the long sought population of cold and dense aggregations with the potential of harbouring the earliest stages of massive star formation. Up to now there has been no systematic study on the temperature distribution, velocity fields, chemical and physical state toward this new cloud population.

Aims. Knowing these properties is crucial for understanding the presence, absence and the very potential of star formation. The present paper aims at addressing these questions. We analyse temperature structures and velocity fields and gain information on their chemical evolution.

Methods. We mapped the $(J, K)=(1,1)$ and $(2,2)$ inversion transitions of ammonia in 9 infrared dark clouds. Our observations allow the most reliable determination of gas temperatures in IRDCs to date.

Results. The gas emission is remarkably coextensive with the extinction seen at infrared wavelengths and with the submillimeter dust emission. Our results show that IRDCs are on average cold $(T<20 \mathrm{~K})$ and have variations among the different cores. IRDC cores are in virial equilibrium, are massive $\left(M>100 M_{\odot}\right)$, highly turbulent $\left(1-3 \mathrm{~km} \mathrm{~s}^{-1}\right)$ and exhibit significant velocity structure (variations around $1-2 \mathrm{~km} \mathrm{~s}{ }^{-1}$ over the cloud).

Conclusions. We find an increasing trend in temperature from IRDCs with high ammonia column density to high mass protostellar objects and Ultracompact HII regions, stages of early warm high-mass star formation. The linewidths of IRDCs are smaller than those observed in high mass protostellar objects and hot core/Ultracompact HII regions. On basis of this sample, and by comparison of the ammonia gas properties within a cloud and between different clouds, we infer that while active star formation is not yet pervasive in most IRDCs, local condensations might collapse in the future or have already begun forming stars.

Key words. stars: formation - ISM: molecules - radio lines: ISM - ISM: kinematics and dynamics

\section{Introduction}

Infrared dark clouds (IRDCs) are cold, dense molecular clouds seen in silhouette against the bright diffuse mid-infrared (MIR) emission of the Galactic plane. They were discovered during mid-infrared imaging surveys with the Infrared Space Observatory (ISO, Perault et al. 1996) and the Mid-course Space Experiment (MSX, Egan et al. 1998).

In an initial census of a $\sim 180^{\circ}$ long strip of the Galactic plane (between $269^{\circ}<l<91^{\circ}, b= \pm 0.5^{\circ}$ ), Egan et al. (1998) found $\sim 2000$ compact objects seen in absorption against bright mid-infrared emission from the Galactic plane. Examination of 2MASS, MSX and IRAS images of these objects reveals that they appear as shadows at all these wavelengths, although they are best identified in the $8.3 \mu \mathrm{m} \mathrm{MSX}$ band, because, first, the 7.7 and $8.6 \mu \mathrm{m}$ PAH features associated with star-forming regions contribute to a brighter background emission and, second, the MSX $8.3 \mu \mathrm{m}$ band is more sensitive than the satellite's other bands. Recently, we have reviewed the observational studies on IRDCs (Menten et al. 2005).
While low mass clouds have been relatively well studied, our picture of high-mass star formation remains unclear (see Evans et al. 2002). IRDCs appear to be the long sought population of cold and dense aggregations with the potential of harbouring the earliest stages of massive star formation. It is likely that some of the stars forming in them are massive (luminosities of submm condensations range up to $10^{4} L_{\odot}$ ). A recent study by Ormel et al. (2005) on an IRDC towards the W51 Giant Molecular Cloud (GMC) suggests that sources of $\sim 300 M_{\odot}$ are embedded within the cores, most likely protostars. Recently, we (Pillai et al. 2005) reported a detailed study of the strongest submm peak in the IRDC G11.11-0.12, where we find clear evidence of a heavily embedded protostar.

The salient results on IRDCs are summarised below. The IRDCs observed so far have sizes of 1-10 pc and have mostly a filamentary morphology. On the basis of LVG calculations of $\mathrm{mm} \mathrm{H}_{2} \mathrm{CO}$ observations Carey et al. (1998) find that typical IRDCs have gas densities of $n>10^{6} \mathrm{~cm}^{-3}$ and temperatures of $T<20 \mathrm{~K}$. Kinematic distances determined from $\mathrm{H}_{2} \mathrm{CO}$ 
Table 1. List of IRDCs observed in $\mathrm{NH}_{3}(1,1)$ and $(2,2)$.

\begin{tabular}{lllcc}
\hline \hline Name & RA(J2000) & Dec(J2000) & $V_{\text {LSR }}\left[\mathrm{km} \mathrm{s}^{-1}\right]$ & D [kpc] \\
\hline G11.11-0.12 P1 & $18: 10: 29.27$ & $-19: 22: 40.3$ & 29.2 & 3.6 \\
G19.30+0.07 & $18: 25: 56.78$ & $-12: 04: 25.0$ & 26.3 & 2.2 \\
G24.72-0.75 & $18: 36: 21.07$ & $-07: 41: 37.7$ & 56.4 & 1 \\
G24.63+0.15 & $18: 35: 40.44$ & $-07: 18: 42.3$ & 54.2 & 3.6 \\
G28.34+0.06 P1 & $18: 42: 50.9$ & $-04: 03: 14$ & 78.4 & 4.8 \\
G28.34+0.06 P2 & $18: 42: 52.4$ & $-03: 59: 54$ & 78.4 & 4.8 \\
G33.71-0.01 & $18: 52: 53.81$ & $+00: 41: 06.4$ & 104.2 & 7.2 \\
G79.27+0.38 & $20: 31: 59.61$ & $+40: 18: 26.4$ & 1.2 & 1 \\
G79.34+0.33 & $20: 32: 21.803$ & $+40: 20: 08.00$ & 0.1 & 1 \\
G81.50+0.14 & $20: 40: 08.29$ & $+41: 56: 26.4$ & 8.7 & 1.3 \\
\hline
\end{tabular}

Notes: Columns are name, right ascension, declination, LSR velocity, distance. Positions and distances are taken from Carey et al. (1998). The coordinates correspond to the reference positions of the maps.

observations using a standard Galactic rotation curve that ranges between 2.2 and $4.8 \mathrm{kpc}$ (Carey et al. 1998) indicate that the clouds are not local. All observed IRDCs in the sample of Carey et al. (2000) contain 1-4 bright sub-millimeter (submm) dust continuum emission peaks ( $>1 \mathrm{Jy} / 14^{\prime \prime}$ beam at $850 \mu \mathrm{m}$ ) surrounded by an envelope of emission which matches the morphology of the IRDC in mid-infrared extinction. The cores corresponding to the brightest submm peaks have masses of 100 to $1200 M_{\odot}$, except for two clouds in the Cygnus region that have masses around $40 M_{\odot}$.

Hennebelle et al. (2001) in a systematic analysis of the ISOGAL images extracted about 450 IRDCs, for which they derive $15 \mu \mathrm{m}$ optical depths of 1 to 4 . Teyssier et al. (2002) reported that Large Velocity Gradient (LVG) model calculations of $\mathrm{HC}_{3} \mathrm{~N},{ }^{13} \mathrm{CO}$, and $\mathrm{C}^{18} \mathrm{O}$ yield densities larger than $10^{5} \mathrm{~cm}^{-3}$ in the densest parts. The authors claim to find kinetic temperatures between 8 and $25 \mathrm{~K}$ based on $\mathrm{CH}_{3} \mathrm{CCH}$ observations; the higher values being found toward embedded objects, however a detailed analysis is hitherto unpublished.

Observations of molecules in IRDCs so far have concentrated on just a few species. Up to now there has been no systematic study on the temperature distribution, velocity fields, chemical and physical state toward this new cloud population. Knowing these properties is crucial for understanding the presence, absence and the very potential of star formation. The present paper aims at addressing these questions. We have started with a survey of a sample of 9 IRDCs in $(1,1)$ and $(2,2) \mathrm{cm}$ rotational transitions of ammonia $\left(\mathrm{NH}_{3}\right)$. This sample has been studied before by Carey et al. (1998) and Carey et al. (2000) and was selected on the basis of the large extent and high contrast of the IRDCs against the MIR background.

Ammonia has proven to be an important tool in measuring the physical conditions in molecular clouds (Ho \& Townes 1983). Since only the lowest $\mathrm{NH}_{3}$ energy levels are expected to be populated in cool dark clouds $(T<20 \mathrm{~K})$, their physical conditions can be probed using the $(1,1)$ and $(2,2)$ inversion transitions in the metastable $(J, K)$ rotational levels of ammonia. Radiative transitions between different $K$-ladders are forbidden, therefore the lowest levels are populated only via collisions. The optical depth can be determined from the ratio of the hyperfine satellites. Thus, the population of the different levels can be estimated and hence the temperature of the gas determined. In addition, recent chemical models reveal that $\mathrm{NH}_{3}$ (and also $\mathrm{N}_{2} \mathrm{H}^{+}$), does not deplete from the gas phase for the densities observed in IRDCs $\left(<10^{6} \mathrm{~cm}^{-3}\right)$ (Bergin \& Langer 1997). Thus $\mathrm{NH}_{3}$ is an excellent tracer of the dense gas where many other molecules would have heavily depleted.

In Sect. 2, we give details of our observations with the Effelsberg $100 \mathrm{~m}$ telescope. In Sect. 3, we discuss the data reduction and present the correlation between gas emission and MIR absorption. In Sect. 4, we derive the rotational temperature, gas kinetic temperature and $\mathrm{NH}_{3}$ column density. Furthermore, we analyse the velocity structure, estimate dust mass, virial mass and $\mathrm{NH}_{3}$ abundance. In Sect. 5, we compare the core gas properties (temperature, $\mathrm{NH}_{3}$ linewidths and column density) with that of other populations of objects that are thought to trace the early stages of high mass star formation. We do a similar comparison with local dark clouds well-studied in $\mathrm{NH}_{3}$. Finally, we speculate on a possible formation mechanism of IRDCs involving supernova remnants.

\section{Observations}

We mapped the IRDCs listed in Table 1 with the Effelsberg $100 \mathrm{~m}$ telescope of the Max-Planck-Institut für Radioastronomie in October 1999. The frontend was the facility $1.3 \mathrm{~cm}$ maser receiver tuned to a frequency of $23.7 \mathrm{GHz}$ centered between the $\mathrm{NH}_{3}(1,1)$ and $(2,2)$ transitions. The spectrometer was a 8192 channel auto-correlator used with 2 subunits of $20 \mathrm{MHz}$ bandwidth each. The resulting spectral resolution was $\approx 0.2 \mathrm{~km} \mathrm{~s}^{-1}$ after smoothing the data to increase the signal-to-noise ratio. The beamwidth at the frequencies of the $\mathrm{NH}_{3}$ lines is $40^{\prime \prime}(F W H M)$. The observations were conducted in frequency switching mode with a frequency throw of $7.5 \mathrm{MHz}$. The maps toward all sources covered the extinction seen in the MSX images and were made with half power beamwidth $(H P B W)$ spacing $\left(40^{\prime \prime}\right)$. Alternate scans were interspaced at half the full beamwidth resulting in $\approx 20 \times \sqrt{(2)}$ spacing. Pointing was checked at roughly hourly intervals by means of continuum drift scans on nearby pointing sources. We found the pointing to be accurate to within $12^{\prime \prime}$.

Absolute calibration is not an issue in determining the rotational temperature, because it is solely governed by the ratio of the $\mathrm{NH}_{3}(1,1)$ hyperfine lines and the $(1,1)$ and $(2,2)$ brightness 
temperatures. However, in order to estimate the excitation temperature and the column density the data needs to be calibrated. The calibration procedure is documented on our webpage ${ }^{1}$. The important steps are mentioned below. The $100 \mathrm{~m}$ data (normally in CLASS format) is in arbitrary noise tube units $\left(T_{\mathrm{N}}\right)$ and has to be converted to main beam brightness temperature units. We observe a standard flux calibrator with known flux $S_{\lambda}$ at wavelength $\lambda$ and thus estimate the conversion factor from $T_{\mathrm{N}}$ scale to Jy.

The main beam brightness temperature $T_{\mathrm{MB}}$ of the calibrator for a given beamwidth $\theta$ and wave length $\lambda$ is then given by

$\left(T_{\mathrm{MB}} / \mathrm{K}\right)=\frac{\left(S_{\lambda} / \mathrm{Jy}\right) \times(\lambda / \mathrm{cm})^{2}}{(\theta / \mathrm{arcmin})^{2} \times 2.65}$.

The primary flux calibration is based upon continuum scans of NGC7027 assuming a flux density of $\approx 5.6 \mathrm{Jy}$ (Ott et al. 1994). The Ultracompact HII (UCHII) regions G10.62-0.38 and G34.26+0.15 and the quasar J1743-038 were used as secondary calibrators which span the entire elevation range and an elevation-dependant calibration factor was derived. The maximum rms uncertainty in calibration for elevations lower than $19^{\circ}$ is $15 \%$.

\section{Data reduction and analysis}

The spectra were reduced using the CLASS package (Forveille et al. 1989). For a given source, the spectra were averaged and a polynomial baseline of order 3-5 subtracted.

The maps were generated by convolving the original data using a Gaussian function with a $H P B W$ of $40^{\prime \prime}$. Table 1 reports the coordinates of the central position for each map.

MSX $8 \mu \mathrm{m}$ images of these clouds with superimposed contours of the $\mathrm{NH}_{3}(1,1)$ integrated intensity are shown in Fig. 1. The velocity range used for integration is within $\pm 25 \mathrm{~km} \mathrm{~s}^{-1}$ of the "Local Standard of Rest Velocity", $V_{\mathrm{LSR}}$, hence including the satellite lines. The $\mathrm{NH}_{3}$ maps of G79.27+0.38 and G33.710.01 have clumpy structures. Some of the clumps may be artificial and caused by the low signal-to-noise ratio.

The reduced $(1,1)$ and $(2,2)$ spectra for the $\mathrm{NH}_{3}$ peak positions of the observed sources are shown in Fig. 2. $\mathrm{NH}_{3}(1,1)$ observations were reduced using "METHOD NH3(1, 1$)$ " in CLASS to fit the hyperfine structure and derive optical depths and linewidths. The standard procedure to analyse the $\mathrm{NH}_{3}$ $(1,1)$ and $(2,2)$ lines have been described in detail by Bachiller et al. (1987).

The hyperfine structure of the $(2,2)$ line is too weak to be observed; therefore, the optical depth could be obtained only for $\mathrm{NH}_{3}(1,1)$. The $\mathrm{NH}_{3}(1,1)$ and $(2,2)$ main beam brightness temperatures are obtained by fitting the main line with a single Gaussian. The $(1,1)$ and $(2,2)$ linewidth is obtained by the hyperfine fitting which accounts for the line broadening due to optical depth effects excluding self-absorption. The parameters obtained from the fits to the $(1,1)$ hyperfine lines are listed in Table 2.

\footnotetext{
${ }^{1}$ www.mpifr-bonn.mpg.de/staff/tpillai/ eff_calib/index.html
}

The basic physical parameters, namely the excitation temperature, rotational temperature, the kinetic temperature and ammonia column density, have been derived using the standard formulation for $\mathrm{NH}_{3}$ spectra (Ho \& Townes 1983). Table 3 summarises the estimates of these parameters toward the cores. We give the formal errors $(1 \sigma)$, derived from Gaussian error propagation.

\section{Results}

Our sample consists of both "Infrared dark clouds", with extents of $\sim 1-10 \mathrm{pc}$, and "Infrared dark cloud complexes", which are comprised of multiple individual clouds. IRDC G11.110.12 would thus be a complex ( $>4 \mathrm{pc}$ ) while $\mathrm{G} 24.63+0.15$ would be a dark cloud. The IRDCs G79.34+0.33 and G79.27+0.38 are essentially two parts of the same extended filament in the Cygnus-X region connected by a bright patch of dust emission as seen in SCUBA and MSX images (Redman et al. 2003).

\subsection{Cloud morphology}

There is generally a close match between the ammonia emission and the mid-infrared extinction as shown in Fig. 1. In G24.72-0.75 and G79.34+0.33, the $\mathrm{NH}_{3}$ emission peak is however correlated with bright and compact MIR emission. Redman et al. (2003) find that the MIR emission in G79.34+0.33 corresponds to a luminous "Young Stellar Object" (YSO) with a strong IR excess which might be interacting with the foreground IRDC. No such interaction has been reported for the IRDC G24.72-0.75 and the nature of the MIR object is unknown.

In general, the cloud geometry is extended and filamentary and in no case close to spherical. The mean aspect ratio determined by fitting $2 \mathrm{D}$ ellipse to the entire $\mathrm{NH}_{3}$ emitting region is 2.2 and the total extent of the clouds ranges from $0.4-8.3 \mathrm{pc}$. G11.11-0.12 is a filamentary cloud as revealed by the $8 \mu \mathrm{m}$ extinction and the $850 \mu \mathrm{m}$ dust emission as shown in Fig. 3. The peaks of the submm emission are strikingly coincident with the ammonia cores. The $\mathrm{NH}_{3}$ map reveals at least two peaks toward the north-east segment and another set of peaks towards the southwest extension of the filament suggesting that there are several unresolved sub-structures or cores within the extended filament.

The strongest submm emission peak P2 in G28.34 is in the close vicinity of the IRAS source 18402-0403. But the peak of the ammonia emission for the northern extension seems to be offset from that of the IRAS source (see Fig. 4). This could be due to the interaction of the IRAS source with the cloud.

\subsection{Source sizes}

Due to the large distances to the clouds, the compact structures within the cloud are marginally resolved with the $40^{\prime \prime}$ beam of our $\mathrm{NH}_{3}$ observations. Hence, estimating the size of the core from $\mathrm{NH}_{3}$ might only deliver upper limits of the order of the beam size. We use channel maps to solve the problem of identifying clumps. The information in the third dimension enables 

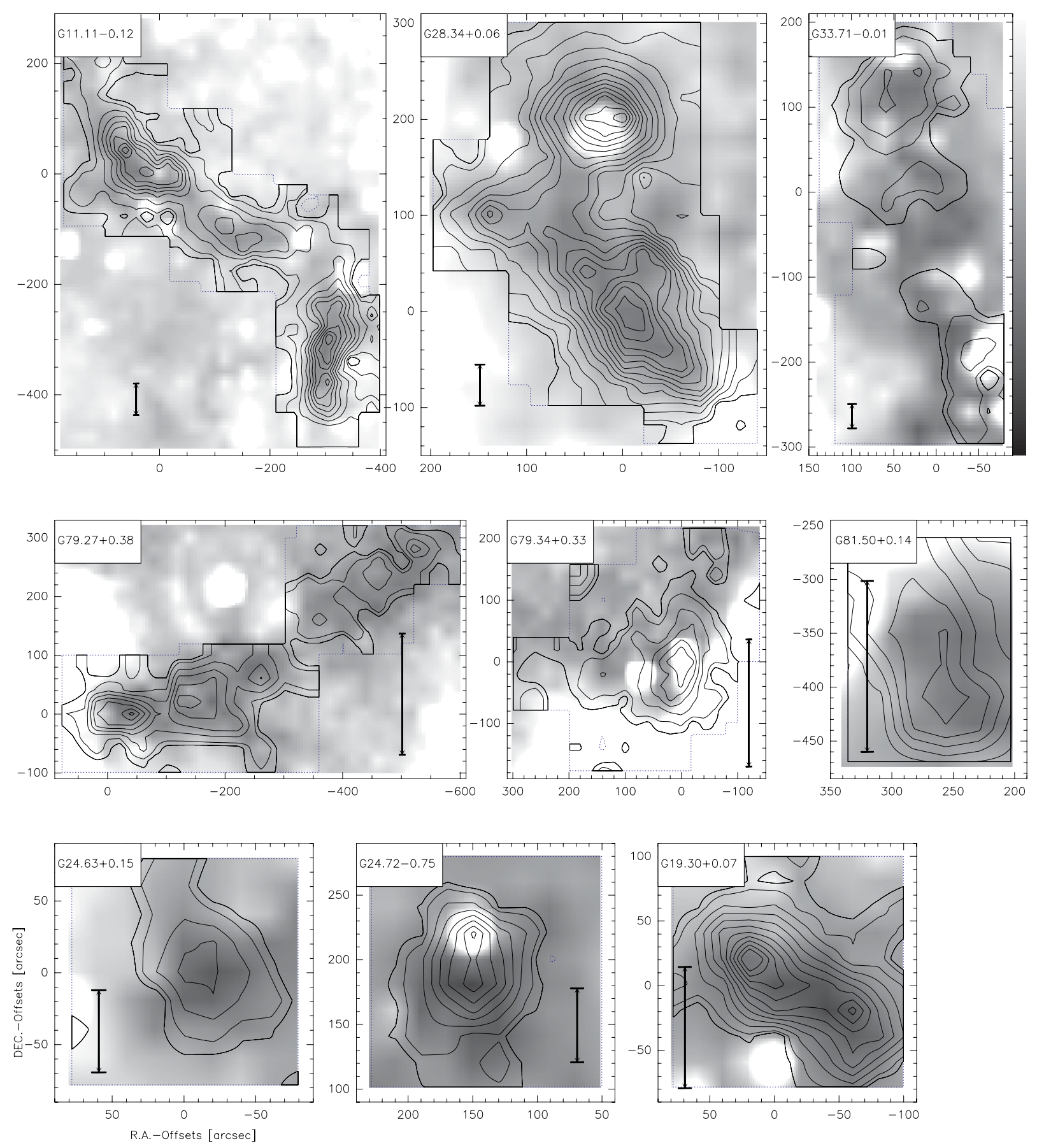

Fig. 1. MSX image of the clouds at $8.3 \mu \mathrm{m}$ (greyscale) with $\mathrm{NH}_{3}(1,1)$ integrated intensity as contours. The $\mathrm{NH}_{3}(1,1)$ maps have a resolution of $40^{\prime \prime}$ and the same spacing. The greyscale corresponds to intensity range shown by the wedge in the upper right corner (from dark to light). The contour levels are $-2 \sigma, 1 \sigma, 2 \sigma, 4 \sigma, 6 \sigma \ldots$ The rms noise levels are given in Table 2 . The approximate map boundary is also indicated. Tick marks are coordinate offsets (in arcseconds) relative to the positions given in Table 1 . The bar indicates a length of $1 \mathrm{pc}$ at the distance of each IRDC.

us to disentangle clumps that are sufficiently well separated in velocity. For those sources with SCUBA $850 \mu \mathrm{m}$ data, we cross-identify the clumps within half the $\mathrm{NH}_{3}$ beam $\left(\sim 20^{\prime \prime}\right)$ at the higher SCUBA resolution $\left(\sim 14^{\prime \prime}\right)$ and estimate the source size by a 2D Gaussian fit to the clumps in the SCUBA data. In cases where we do not have dust emission maps, we use the 2D Gaussian fit routine in GRAPHIC. It searches for the brightest pixel in each velocity channel of the channel map across the $\mathrm{NH}_{3}(1,1)$ main component and fits a 2D Gaussian to determine source size. The output is then cross-checked over the different channels and the fit obtained for the brightest emission is used. We find 1-5 clumps for each cloud. The source sizes after correcting for the beam size (after subtracting the Gaussian beam size in quadrature) are listed in Table 3. The dense cores within these IRDCs are thus not resolved with the $40^{\prime \prime} 100 \mathrm{~m}$ beam. We find several secondary peaks offset 

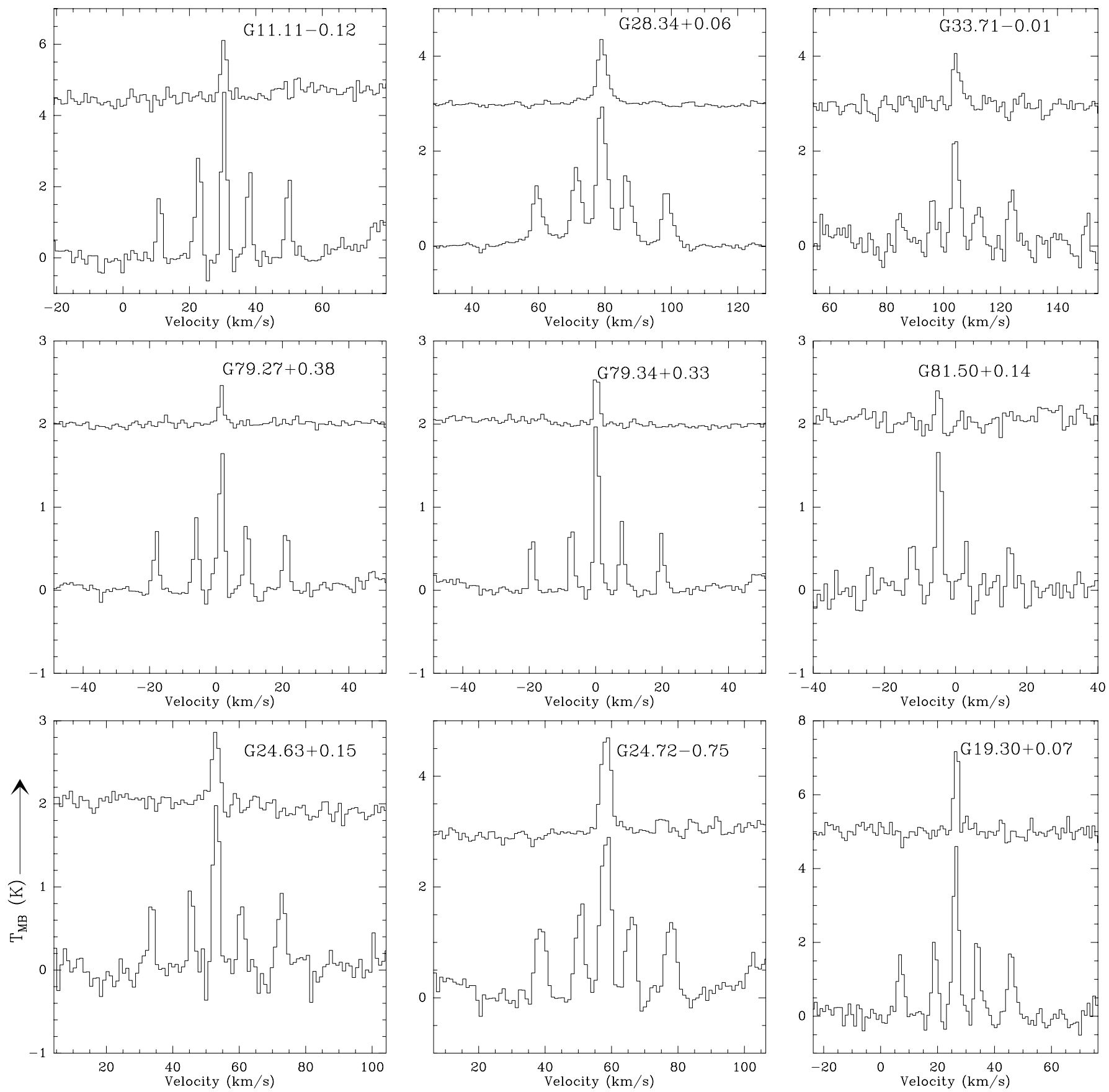

Fig. 2. $\mathrm{NH}_{3}(1,1)$ (lower histogram in each panel) and (2, 2) (upper histogram, offset from $0 \mathrm{~K}$ for clarity) spectra toward the brightest position of the 9 observed sources. The brightest positions are referred to as $\mathrm{NH}_{3} \mathrm{P} 1$ in Table 3 .

by $>1 \sigma$ in most sources; offsets from the central position are given in Table 3. Table 3 should be referred to for the nomenclature of the clumps identified in the $\mathrm{NH}_{3}$ maps and/or the SCUBA $850 \mu \mathrm{m}$ images hereafter.

\subsection{Line profiles}

In general, $\mathrm{NH}_{3}$ lines are brighter than the mm rotational $\mathrm{H}_{2} \mathrm{CO}$ transitions observed by Carey et al. (1998). As given in Table 2 , the $(2,2)$ peak line intensity is on average $40 \%$ of that of the $(1,1)$ line. Gaussian line profiles with extended wings have been reported for $\mathrm{H}_{2} \mathrm{CO}$ lines in most of the clouds
(Carey et al. 1998) but we do not observe any pronounced wings in $\mathrm{NH}_{3}$. Leurini et al. (2006, submitted) find line profiles similar to those observed in $\mathrm{H}_{2} \mathrm{CO}$ in the mm transitions of $\mathrm{CH}_{3} \mathrm{OH}$.

\subsection{Linewidth}

The width of a spectral line is an important parameter because it is a measure of the total kinetic energy of the cloud. The $\mathrm{NH}_{3}(1,1)$ linewidths for all sources lie between $0.8-3 \mathrm{~km} \mathrm{~s}^{-1}$. The $\mathrm{H}_{2} \mathrm{CO}$ linewidths are significantly larger (factor 2) than the $\mathrm{NH}_{3}$ linewidths in all cases except $\mathrm{G} 81.50+0.14$, where the $\mathrm{NH}_{3}$ linewidths are twice as large. 
Table 2. $\mathrm{NH}_{3}(1,1)$ and $(2,2)$ map results: peak position.

\begin{tabular}{|c|c|c|c|c|c|c|}
\hline Source & Transition & $\begin{array}{l}V_{\mathrm{LSR}} \\
{\left[\mathrm{km} \mathrm{s}^{-1}\right]}\end{array}$ & $\begin{array}{l}T_{\mathrm{MB}} \\
{[\mathrm{K}]}\end{array}$ & $\begin{array}{l}F W H M \\
{\left[\mathrm{~km} \mathrm{~s}^{-1}\right]}\end{array}$ & $\tau_{\text {main }}$ & $\begin{array}{l}\sigma \sigma \\
{\left[\mathrm{K} \mathrm{km} \mathrm{s}^{-1}\right]}\end{array}$ \\
\hline \multirow[t]{2}{*}{ G11.11-0.12 $\mathrm{NH}_{3} \mathrm{P} 1$} & $1-1$ & $30.44( \pm 0.02)$ & $5.47( \pm 0.96)$ & $1.27( \pm 0.06)$ & $3.52( \pm 0.39)$ & 0.42 \\
\hline & $2-2$ & $30.42( \pm 0.09)$ & $1.97( \pm 0.32)$ & $1.56( \pm 0.30)$ & & \\
\hline \multirow[t]{2}{*}{$\mathrm{G} 19.30+0.07 \mathrm{NH}_{3} \mathrm{P} 1$} & $1-1$ & $26.45( \pm 0.03)$ & $5.17( \pm 0.8)$ & $1.88( \pm 0.08)$ & $1.88( \pm 0.26)$ & 0.53 \\
\hline & $2-2$ & $26.64( \pm 0.06)$ & $2.86( \pm 0.27)$ & $1.57( \pm 0.17)$ & & \\
\hline \multirow[t]{2}{*}{$\mathrm{G} 24.72-0.75 \mathrm{NH}_{3} \mathrm{P} 1$} & $1-1$ & $58.37( \pm 0.07)$ & $2.76( \pm 0.45)$ & $2.57( \pm 0.12)$ & $2.81( \pm 0.47)$ & 0.25 \\
\hline & $2-2$ & $58.47( \pm 0.12)$ & $1.99( \pm 0.23)$ & $2.97( \pm 0.24)$ & & \\
\hline \multirow[t]{2}{*}{$\mathrm{G} 24.63+0.15$} & $1-1$ & $53.11( \pm 0.04)$ & $2.40( \pm 0.34)$ & $1.7( \pm 0.09)$ & $2.36( \pm 0.43)$ & 0.16 \\
\hline & $2-2$ & $52.89( \pm 0.09)$ & $0.98( \pm 0.12)$ & $2.42( \pm 0.24)$ & & \\
\hline \multirow[t]{2}{*}{$\mathrm{G} 28.34+0.06 \mathrm{NH}_{3} \mathrm{P} 1$} & $1-1$ & $79.14( \pm 0.02)$ & $2.88( \pm 0.42)$ & $2.67( \pm 0.04)$ & $2.00(0.11)$ & 0.16 \\
\hline & $2-2$ & $79.15( \pm 0.04)$ & $1.37( \pm 0.05)$ & $3.29( \pm 0.11)$ & & \\
\hline \multirow[t]{2}{*}{ G33.71-0.01 NH $\mathrm{N}_{3} \mathrm{P} 1$} & $1-1$ & $104.31( \pm 0.09)$ & $2.52( \pm 0.43)$ & $2.64( \pm 0.2)$ & $1.5( \pm 0.52)$ & 0.12 \\
\hline & $2-2$ & $104.4( \pm 0.17)$ & $1.14( \pm 0.22)$ & $2.40( \pm 0.35)$ & & \\
\hline \multirow[t]{2}{*}{$\mathrm{G} 79.27+0.38 \mathrm{NH}_{3} \mathrm{P} 1$} & $1-1$ & $1.65( \pm 0.02)$ & $2.28( \pm 0.38)$ & $1.04( \pm 0.05)$ & $2.49( \pm 0.27)$ & 0.31 \\
\hline & $2-2$ & $1.63( \pm 0.05)$ & $0.61( \pm 0.08)$ & $0.98( \pm 0.14)$ & & \\
\hline \multirow[t]{2}{*}{ G79.34+0.33 $\mathrm{NH}_{3} \mathrm{P} 1$} & $1-1$ & $0.31( \pm 0.01)$ & $2.53( \pm 0.30)$ & $1.23( \pm 0.03)$ & $1.45( \pm 0.13)$ & 0.19 \\
\hline & $2-2$ & $0.25( \pm 0.04)$ & $0.79( \pm 0.07)$ & $1.29( \pm 0.13)$ & & \\
\hline \multirow[t]{2}{*}{ G81.50+0.14 } & $1-1$ & $-4.53( \pm 0.05)$ & $2.21( \pm 0.35)$ & $1.35( \pm 0.15)$ & $0.37( \pm 0.46)$ & 0.27 \\
\hline & $2-2$ & $-4.69( \pm 0.12)$ & $0.67( \pm 0.21)$ & $1.00( \pm 0.34)$ & & \\
\hline
\end{tabular}

Notes: Columns are name, $\mathrm{NH}_{3}(J, K)$ transition, LSR velocity, $(1,1)$ main beam brightness temperature, full linewidth at half maximum, main group optical depth and the 1 sigma noise level in the $\mathrm{NH}_{3}(1,1)$ integrated intensity map (1). The error estimates are given in brackets.

Table 3. Physical properties of observed IRDCs.

\begin{tabular}{|c|c|c|c|c|c|c|c|c|c|c|c|c|}
\hline Source & $\begin{array}{c}\text { Offsets } \\
\text { [arcsecs] }\end{array}$ & $\begin{array}{l}T_{\mathrm{EX}} \\
{[\mathrm{K}]}\end{array}$ & $\begin{array}{r}T_{\mathrm{ROT}} \\
{[\mathrm{K}]}\end{array}$ & $\begin{array}{r}T_{\mathrm{KIN}} \\
{[\mathrm{K}]}\end{array}$ & $\begin{array}{r}N\left(\mathrm{NH}_{3}\right) \\
{\left[10^{14} \mathrm{~cm}^{-} 2\right]}\end{array}$ & $\begin{array}{c}N\left(\mathrm{H}+\mathrm{H}_{2}\right) \\
{\left[10^{22} \mathrm{~cm}^{-} 2\right]}\end{array}$ & $\begin{array}{l}\chi_{\mathrm{NH}_{3}} \\
{\left[10^{-8}\right]}\end{array}$ & $\begin{array}{c}\text { Mass } \\
M_{\odot}\end{array}$ & $\begin{array}{c}\text { Size } \\
{[\operatorname{arcsecs}]}\end{array}$ & $\begin{array}{c}M_{\mathrm{vir}} \\
{\left[M_{\odot}\right]}\end{array}$ & $\alpha$ & $\begin{array}{c}M_{\mathrm{NH}_{3}} \\
{\left[10^{4} M_{\odot}\right]}\end{array}$ \\
\hline G11.11-0.12 $\mathrm{NH}_{3} \mathrm{P} 1$ & $(80,40)$ & $7.6( \pm 0.7)$ & $12.3( \pm 1.2)$ & 12.7 & $41.5( \pm 6.4)$ & 8.4 & 4.9 & 485 & 27 & 468 & 1.0 & 18.1 \\
\hline G11.11-0.12 $\mathrm{NH}_{3} \mathrm{P} 2$ & $(0,0)$ & $6.6( \pm 0.4)$ & $13.4( \pm 1.0)$ & 13.8 & $30.5( \pm 3.3)$ & 10.2 & 2.9 & 172 & 13 & 268 & 1.6 & \\
\hline G11.11-0.12 $\mathrm{NH}_{3} \mathrm{P} 3$ & $(-300,-325)$ & $7.1( \pm 0.3)$ & $14.1( \pm 9.7)$ & 14.5 & $60.4( \pm 11.4)$ & 8.7 & 6.9 & 647 & 32 & 860 & 1.3 & \\
\hline G11.11-0.12 $\mathrm{NH}_{3} \mathrm{P} 4$ & $(-300,-400)$ & $5.7( \pm 0.5)$ & $10.5( \pm 1.0)$ & 10.8 & $50.9( \pm 10.6)$ & 16.1 & 3.2 & 788 & 25 & 555 & 0.7 & \\
\hline $\mathrm{G} 19.30+0.07 \mathrm{NH}_{3} \mathrm{P}^{n}$ & $(20,20)$ & $8.2( \pm 0.9)$ & $17.6( \pm 2.0)$ & 18.4 & $25.9( \pm 3.2)$ & & & & 38 & 893 & & 0.9 \\
\hline $\mathrm{G} 19.30+0.07 \mathrm{NH}_{3} \mathrm{P} 2^{n}$ & $(-60,-20)$ & $5.9( \pm 0.7)$ & $13.8( \pm 1.8)$ & 14.3 & $33.3( \pm 6.9)$ & & & & 45 & 823 & & \\
\hline $\mathrm{G} 24.72-0.17 \mathrm{NH}_{3} \mathrm{P} 1^{n}$ & $(150,220)$ & $6.6( \pm 0.6)$ & $17.4( \pm 2.2)$ & 18.2 & $44.3( \pm 5.7)$ & & & & 34 & 2450 & & 1.4 \\
\hline $\mathrm{G} 24.63+0.15^{n}$ & $(0,0)$ & $5.0( \pm 0.5)$ & $14.3( \pm 1.4)$ & 14.8 & $24.1( \pm 4.4)$ & & & & 43 & 1358 & & 0.7 \\
\hline $\mathrm{G} 28.34+0.06 \mathrm{NH}_{3} \mathrm{P} 1$ & $(0,0)$ & $5.7( \pm 0.2)$ & $16.0( \pm 1.2)$ & 16.6 & $32.1( \pm 2.2)$ & 5.8 & 5.5 & 904 & 22 & 2280 & 2.5 & 25.4 \\
\hline $\mathrm{G} 28.34+0.06 \mathrm{NH}_{3} \mathrm{P} 2$ & $(20,200)$ & $5.5( \pm 0.2)$ & $15.4( \pm 1.4)$ & 16.0 & $38.6( \pm 3.2)$ & 32.7 & 1.2 & 2310 & 45 & 2928 & 1.3 & \\
\hline $\mathrm{G} 28.34+0.06 \mathrm{NH}_{3} \mathrm{P} 3$ & $(-60,-60)$ & $4.8( \pm 0.2)$ & $12.8( \pm 1.2)$ & 13.2 & $47.2( \pm 6.1)$ & 5.9 & 8 & 374 & 21 & 1398 & 3.7 & \\
\hline $\mathrm{G} 28.34+0.06 \mathrm{NH}_{3} \mathrm{P} 4$ & $(-30,50)$ & $4.8( \pm 0.2)$ & $15.9( \pm 2.2)$ & 16.4 & $37.4( \pm 4.9)$ & 3.7 & 10.1 & 147 & 16 & 960 & 6.5 & \\
\hline $\mathrm{G} 28.34+0.06 \mathrm{NH}_{3} \mathrm{P} 5$ & $(50,40)$ & $5.6( \pm 0.3)$ & $13.6( \pm 1.3)$ & 14.1 & $29.6( \pm 3.8)$ & 6.3 & 4.7 & 528 & 24 & 1378 & 2.6 & \\
\hline $\mathrm{G} 33.71-0.01 \mathrm{NH}_{3} \mathrm{P} 1{ }^{n}$ & $(-40,-200)$ & $5.6( \pm 1.1)$ & $16.6( \pm 2.3)$ & 17.2 & $23.0( \pm 6.6)$ & & & & 52 & 7880 & & 15.6 \\
\hline $\mathrm{G} 33.71-0.01 \mathrm{NH}_{3} \mathrm{P} 2^{n}$ & $(60,100)$ & $5.3( \pm 0.3)$ & $17.1( \pm 2.0)$ & 17.8 & $29.0( \pm 3.3)$ & & & & 60 & 4928 & & \\
\hline $\mathrm{G} 79.27+0.38 \mathrm{NH}_{3} \mathrm{P} 1$ & $(-20,0)$ & $4.8( \pm 0.2)$ & $12.4( \pm 0.9)$ & 12.8 & $20.5( \pm 2.5)$ & 14.2 & 1.4 & 84 & 32 & 155 & 1.8 & 0.4 \\
\hline $\mathrm{G} 79.27+0.38 \mathrm{NH}_{3} \mathrm{P} 2$ & $(-140,40)$ & $6.0( \pm 0.7)$ & $11.2( \pm 1.4)$ & 11.5 & $11.3( \pm 2.7)$ & 12.1 & 0.9 & 55 & 25 & 53 & 1.0 & \\
\hline $\mathrm{G} 79.27+0.38 \mathrm{NH}_{3} \mathrm{P} 3$ & $(-250,70)$ & $3.3( \pm 2.2)$ & $11.7( \pm 1.6)$ & 12.0 & $10.0( \pm 25.0)$ & 6.5 & 1.5 & 19 & 20 & 40 & 2.1 & \\
\hline $\mathrm{G} 79.34+0.33 \mathrm{NH}_{3} \mathrm{P} 1$ & $(0,0)$ & $5.8( \pm 0.3)$ & $14.1( \pm 0.8)$ & 14.6 & $11.9( \pm 1.0)$ & 17.5 & 0.7 & 143 & 39 & 178 & 1.2 & 0.2 \\
\hline $\mathrm{G} 81.50+0.14^{n}$ & $(260,-375)$ & $5.6( \pm 1)$ & $14.9( \pm 1.3)$ & 15.4 & $7.6( \pm 2.0)$ & & & & 78 & 570 & & 0.1 \\
\hline
\end{tabular}

Notes: Columns are name, offset position from reference, excitation temperature, $\mathrm{NH}_{3}$ rotational temperature, kinetic temperature, $\mathrm{NH}_{3}$ column density, $\mathrm{H}_{2}$ column density, $\mathrm{NH}_{3}$ abundance, gas mass estimated from SCUBA data, size of the cores, virial masses from $\mathrm{NH}_{3}$ the alpha parameter defined as in total cloud mass from $\mathrm{NH}_{3}$. The formal errors are given in brackets. ${ }^{n}$ are those sources for which the sizes have been derived from $\mathrm{NH}_{3}$ data. The $\mathrm{H}_{2}$ column densities are estimated from $850 \mu \mathrm{m}$ SCUBA map (Carey et al. 2000).

The $\mathrm{NH}_{3}$ linewidths for our sample are higher than those of $\mathrm{NH}_{3}$ cores reported in Jijina et al. (1999), which are mostly low mass cores. The large linewidths might be explained as due to clumping. Clumps with smaller linewidth but with a higher clump-to-clump velocity dispersion may add up to the observed linewidths. The sources closest to us (G79 IRDCs) have the smallest linewidth and belong to the Cygnus-X region. In order to test whether the larger linewidth we observe at larger distance is a distance effect, we average the G79 IRDC linewidth over an area $\delta A \propto\left(d_{\text {far }} / d_{\text {Cyg }}\right)^{2}$ where $d_{\text {far }}$ is the distance to a source farther than Cyg and with a much higher linewidth. On comparing the resultant scaled values, we find that the linewidths are still significantly larger for the sources at larger distances after accounting for the larger region in each beam for the more distant cores.

To illustrate this point, let us take the example of the two extreme values of linewidths from our sample. Dark core G79.27+0.38 $\mathrm{NH}_{3} \mathrm{P} 2$ (at $1 \mathrm{kpc}$ ) has a linewidth at the brightest position of $0.83 \mathrm{~km} \mathrm{~s}^{-1}$ while G28.34+0.06 $\mathrm{NH}_{3} \mathrm{P} 2$ (at $4.8 \mathrm{kpc}$ ) has a linewidth of $2.65 \mathrm{~km} \mathrm{~s}^{-1}$. Neither of the two cores have a MIR counterpart which might indicate any deeply embedded protostar influencing its immediate environments. Averaging all the emission in G79.27+0.38 over an area of $\sim 200 \times 200^{\prime \prime}$, we obtain a linewidth of $1.5 \mathrm{~km} \mathrm{~s}^{-1}$, still smaller 


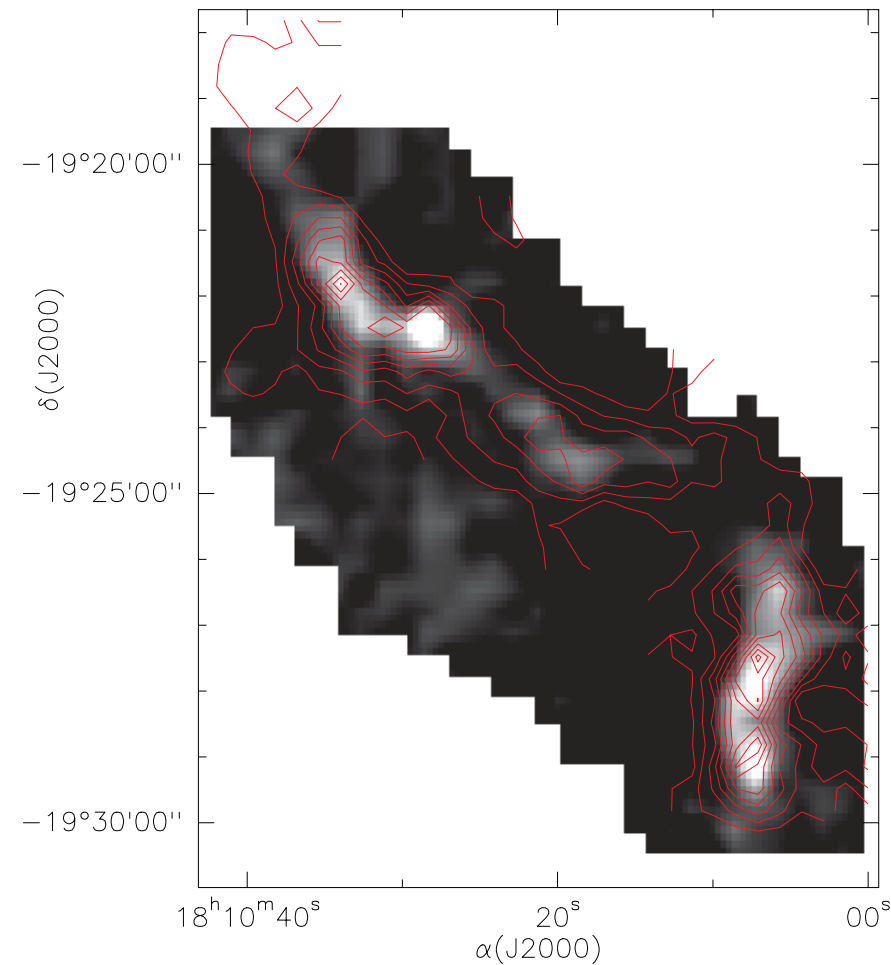

Fig. 3. SCUBA $850 \mu \mathrm{m}$ image (Carey et al. 2000) of the cloud G11.110.12 with $\mathrm{NH}_{3}(1,1)$ integrated intensity as contours. The contour levels are multiples of $2 \sigma$. SCUBA image has a resolution of $14^{\prime \prime}$.

than $2.65 \mathrm{~km} \mathrm{~s}^{-1}$. Therefore, the large linewidths derived appears to be mainly due to the velocity dispersions within the beam.

One of the assumptions made to derive the temperature is that the beam filling factor is the same for both inversion lines; that is, the two lines are emitted by the same volume of gas. The $(2,2)$ linewidths are slightly larger than the $(1,1)$ linewidths (cf. Table 2) toward only some cores, suggesting that the same gas is not exactly traced by both lines in those sources.

The linewidths exhibited by these sources are much larger than the thermal linewidth, $\Delta v_{\text {th }}$, which for $T_{\text {kin }} \sim 20 \mathrm{~K}$ should be $\sim 0.22 \mathrm{~km} \mathrm{~s}^{-1}$ as per the relation

$\Delta v_{\text {th }} \sim \sqrt{\frac{8 \ln (2) k T_{\text {kin }}}{m_{\mathrm{NH}_{3}}}}$,

where $k$ is Boltzmann's constant and $m_{\mathrm{NH}_{3}}$ is the mass of the ammonia molecule. Average linewidths $\sim 2 \mathrm{~km} \mathrm{~s}^{-1}$ could be explained in terms of velocity gradients due to rotation of the cloud, or turbulent cloud movements. The linewidths seems to be especially high towards the cloud G28.34+0.06, where there are several unresolved $\mathrm{NH}_{3}$ clumps, identified in the SCUBA images. In the G79 IRDC the linewidth decreases from G79.34+0.33 $\mathrm{NH}_{3} \mathrm{P} 1\left(1.22 \pm 0.03 \mathrm{~km} \mathrm{~s}^{-1}\right)$ to $\mathrm{G} 79.27+0.38$ $\mathrm{NH}_{3} \mathrm{P} 3\left(0.82 \pm 0.03 \mathrm{~km} \mathrm{~s}^{-1}\right)$. As shown in Fig. 5, we find that the linewidth anti-correlates with optical depth for G28.34 while there is a possible positive correlation G79.27.

G79.27+0.38 $\mathrm{NH}_{3} \mathrm{P} 1$ seems to be more quiescent than its surroundings. In the G79 cloud complex, there is considerable difference in linewidth $\left(\sim 0.5 \mathrm{~km} \mathrm{~s}^{-1}\right)$ between the

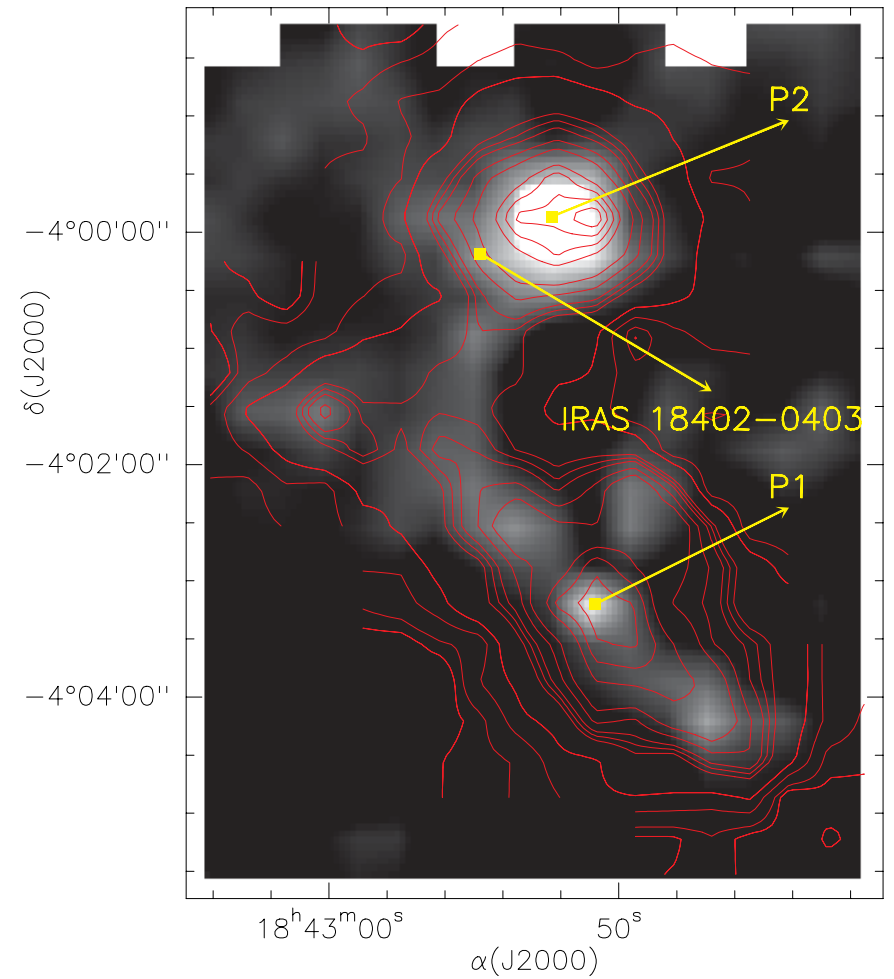

Fig. 4. SCUBA $850 \mu \mathrm{m}$ image of $\mathrm{G} 28.34$ with $\mathrm{NH}_{3}(1,1)$ integrated intensity as contours. The contour levels are $(2 \sigma, 4 \sigma, 6 \sigma \ldots)$.

different clumps along the cloud from east to west. Thus, these clouds might be harbouring objects at different stages of evolution (Redman et al. 2003).

\subsection{Kinetic temperature}

Since the optical depth is known, we derive the excitation temperature of the $\mathrm{NH}_{3}(1,1)$ inversion transition (Table 3 ) via the relation

$T_{\mathrm{EX}}=\frac{T_{\mathrm{MB}}}{1-\mathrm{e}^{-\tau}}+2.7 \mathrm{~K}$,

where $T_{\mathrm{MB}}$ and $\tau$ represents the temperature and the optical depth of the of the $(1,1)$ line. By fitting the main and the hyperfine components of the $(1,1)$ line and the main component of the $(2,2)$ line, we obtain the rotational temperature. An analytical expression (Tafalla et al. 2004) has been used to estimate the kinetic temperature from the rotational temperature. The kinetic temperature is given by the expression

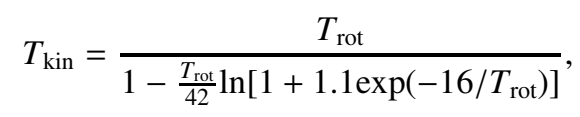

The typical temperatures for the cores range from $11-17 \mathrm{~K}$ as displayed in Table 3 . In some cases, we find that the fit to the $\mathrm{NH}_{3}(1,1)$ line slightly underestimates the peak intensity.

We find that the kinetic temperature is significantly higher than the excitation temperature, as given in Table 3. The beam filling factor $\eta$ is a measure of the fraction of the beam filled by the observed source. Assuming that the cores are in local 

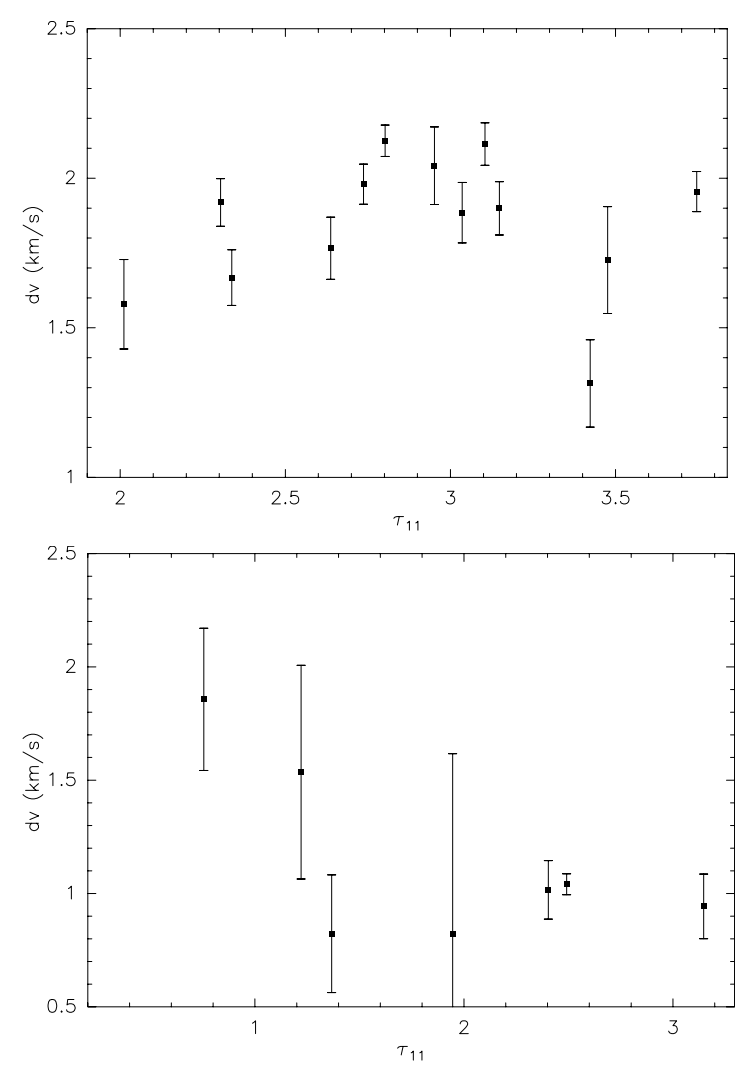

Fig. 5. Correlation plot between the $\mathrm{NH}_{3}(1,1)$ optical depth and the $\mathrm{NH}_{3}(1,1)$ linewidth. Bottom panel: G79.27+0.38 $\mathrm{NH}_{3} \mathrm{P} 1$, Top panel: G28.34+0.06 $\mathrm{NH}_{3} \mathrm{P} 2$.

thermodynamic equlibrium (LTE), we may estimate this fraction as $\eta=T_{\mathrm{EX}} / T_{\text {kin }}$ where $T_{\mathrm{Kin}}$ is the kinetic temperature of the gas. We derive filling factors of $\sim 0.3-0.5$ for all the clumps. These low filling factors suggest either sub-thermal excitation (non LTE conditions) or clumping within the beam. From our estimation of the sizes (Table 3), we find most of the cores are unresolved with the $40^{\prime \prime}$ beam. Thus clumping is more likely to explain the small filling factors.

We find hints of temperature gradients (inside-out) within the cores in three sources and a reverse gradient in one source. However the large error bars associated with the rotational temperature does not allow us to make a convincing case. The temperature structure will be analysed in detail in a future paper with observations at high angular resolution (Pillai et al. 2006 in prep.).

There is also a variation in temperature within the different cores of the same cloud. In G11.11-0.12, with a projected extent of a few pc, the average (over the core size) temperatures of the southern cores are $2-3 \mathrm{~K}(10.9 \pm 1 \mathrm{~K})$ lower than the northern cores $(13.4 \pm 1 \mathrm{~K})$.

In $\mathrm{G} 28.34+0.06$, the gas temperature derived towards $\mathrm{NH}_{3}$ $\mathrm{P} 3,12.7 \pm 1 \mathrm{~K}$ and $\mathrm{NH}_{3} \mathrm{P} 5,13.6 \pm 1 \mathrm{~K}$ is significantly lower than in the rest of the cloud. $\mathrm{CH}_{3} \mathrm{OH}$ observations on the $\mathrm{P} 1$ position (Leurini et al. 2006, submitted) reveal a cold and a hot component, the latter with a small filling factor. We obtain kinetic temperatures of $\sim 16 \mathrm{~K}$ towards the peaks of $\mathrm{NH}_{3} \mathrm{P} 1$ and $\mathrm{NH}_{3} \mathrm{P} 2$. Higher angular resolution observations are needed to confirm a hot component of small extent also in ammonia.

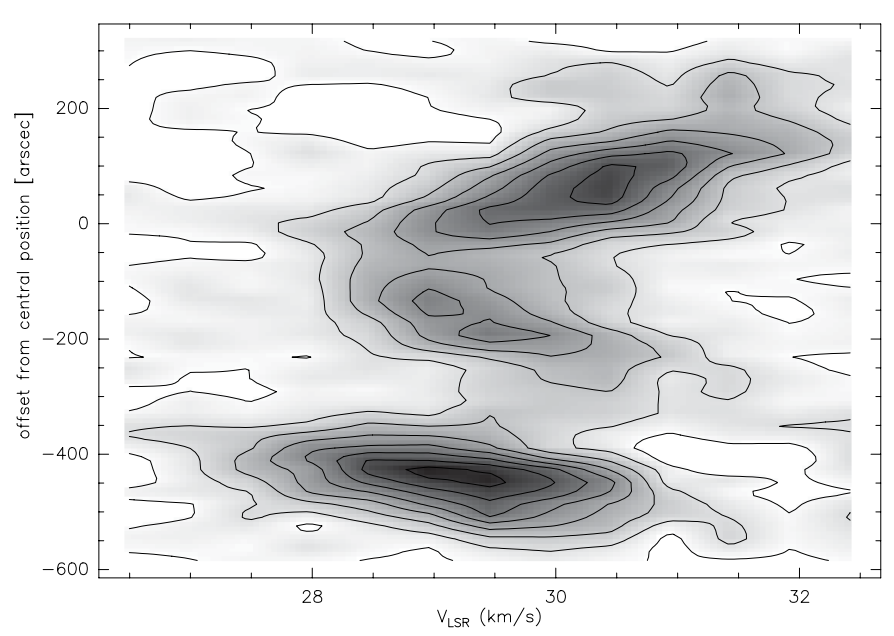

Fig. 6. The average velocity-position map of $\mathrm{NH}_{3}(1,1)$ for the cloud G11.11-0.12 at a position angle of $\sim 40^{\circ}$. Note that the $Y$ axis is the offset from the reference position, given in Table 1. Offsets run from south-west (negative offsets) to north-east (positive offsets).

In G19.30+0.07, the temperature at the position of peak $\mathrm{NH}_{3}$ emission $\mathrm{P} 1$ is $17.9 \pm 1.6 \mathrm{~K}$, much warmer than the rest of the cloud $(13.1 \pm 1.0 \mathrm{~K})$.

In the G79 complex, we find that the temperature in G79.34+0.33 $\mathrm{NH}_{3} \mathrm{P} 1$ is higher $(14.3 \pm 0.8 \mathrm{~K})$ than the other 2 cores $\mathrm{G} 79.27+0.38 \mathrm{NH}_{3} \mathrm{P} 1(12.2 \pm 0.8 \mathrm{~K}) \& \mathrm{G} 79.27+0.38$ $\mathrm{NH}_{3} \mathrm{P} 2(11.1 \pm 1.2 \mathrm{~K})$ in the western part of the filament.

\subsection{Velocity structure}

The average velocity-position diagram along an axis with position angle of $+40^{\circ}$ (to align the position axis roughly with the direction of the filament on the sky) for the cloud G11.11-0.12 is shown in Fig. 6. At every position along the position axis, the $\mathrm{NH}_{3}(1,1)$ spectra were averaged along a line perpendicular to the position axis. There is a clear trend for the velocities to decrease from the south to the P1 position and increase to the north of the NE filament. This is also seen in the channel maps shown in Fig. 7 where the different clumps in the north and south appear at distinctly separate velocities. Given that the $\mathrm{NH}_{3}(1,1)$ linewidth at all positions is $\leq 2.5 \mathrm{~km} \mathrm{~s}^{-1}$, this velocity shift $\left(>5 \mathrm{~km} \mathrm{~s}^{-1}\right)$ between the peaks is certainly significant. The unresolved clump G11.11-0.12 $\mathrm{NH}_{3} \mathrm{P} 4$ is more associated with the G11.11-0.12 $\mathrm{NH}_{3} \mathrm{P} 2$ clump while $\mathrm{P} 3$ fades away at those velocities.

Recently, this filament had been studied in absorption against the diffuse $8 \mu \mathrm{m}$ Galactic background and in emission from cold dust at $850 \mu \mathrm{m}$ (Johnstone et al. 2003). They model the $850 \mu \mathrm{m}$ emission by fitting a non-magnetic isothermal cylinder profile to the radial structure of the entire filament (south and north segment). The velocity structure observed in $\mathrm{NH}_{3}(1,1)$, however, shows several distinct clumps. This suggests that the density structure of the filament cannot be rigorously described by a simple continuous cylinder.

The channel maps for G28.34+0.06 are shown in Fig. 8. The two extensions of the cloud around P1 and P2 differ in velocity by about $1.5 \mathrm{~km} \mathrm{~s}^{-1}$. This is the difference in velocity 


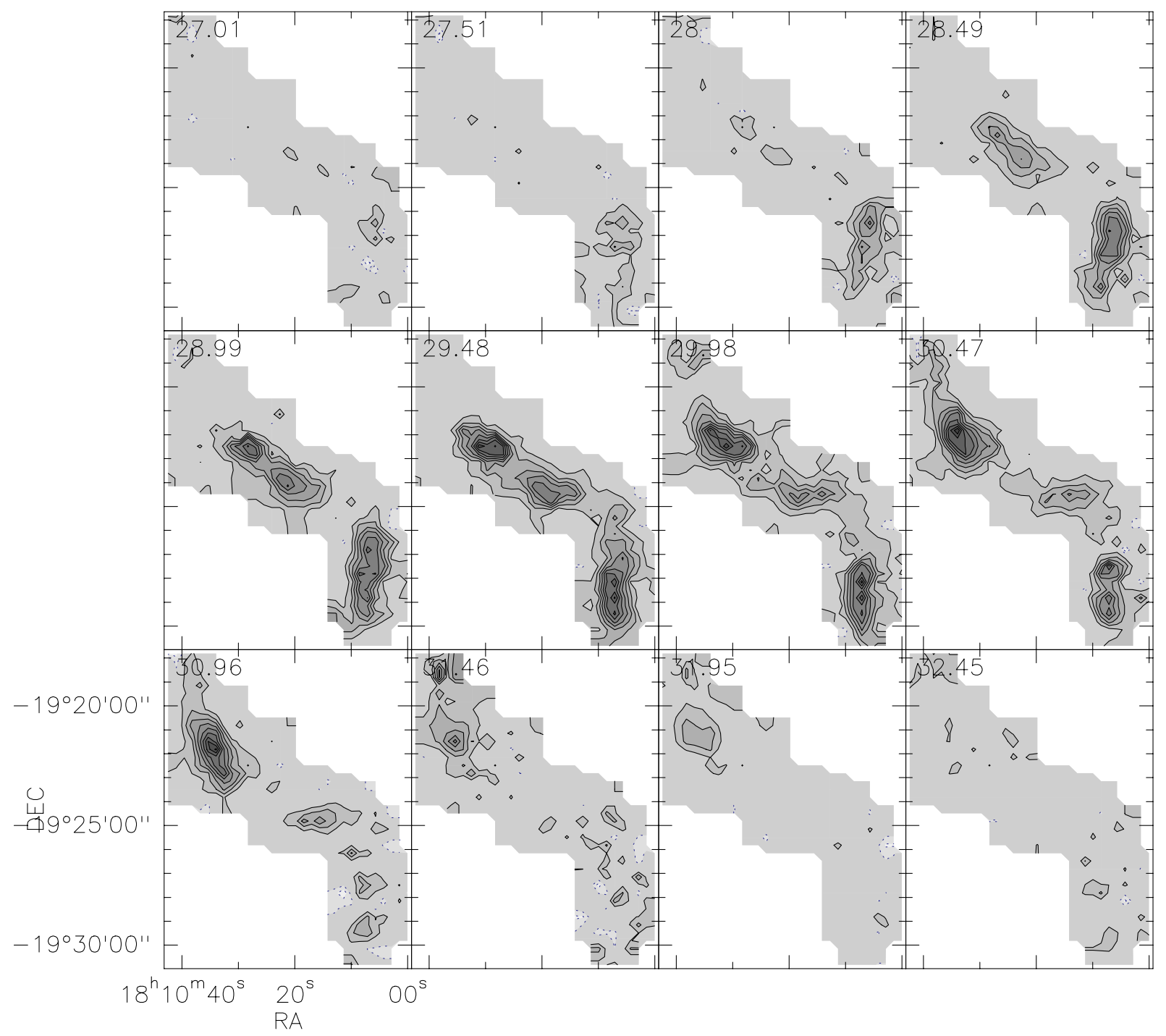

Fig. 7. Channel maps for G11.11-0.12 over the main component for the $\mathrm{NH}_{3}(1,1)$ transition.

for the two peaks at which they are brightest. From the channel maps it appears that there is a bridge between the two parts of the cloud connecting P1 and P2. At the velocity of $80.16 \mathrm{~km} \mathrm{~s}^{-1}$, the three unresolved clumps, which are identified in dust continuum emission but not clearly identified in the $\mathrm{NH}_{3}(1,1)$ integrated intensity emission, are revealed.

G33.71-0.01 shows another interesting case of distinct velocity variations across a cloud. The average velocity position map along the declination axis is displayed in Fig. 9. The velocity increases from the south at $\sim 103 \mathrm{~km} \mathrm{~s}^{-1}$ towards the north to $\sim 106 \mathrm{~km} \mathrm{~s}^{-1}$ while showing a very wide weak component at $\sim 105 \mathrm{~km} \mathrm{~s}^{-1}$ at the $(0,0)$ position. Its detection at $(0,0)$ is only at the $2 \sigma$ level but is also observed in $\mathrm{NH}_{3}(2,2)$. A possible interaction with the nearby SNR G33.6+0.1 is discussed in Sect. 5.2.

\subsection{Column density, $\mathrm{NH}_{3}$ abundance and masses}

We have estimated the column densities and total masses for the bright, compact sources for which the SCUBA $850 \mu \mathrm{m}$ maps are available, after smoothing the data to the resolution of $100 \mathrm{~m}$ beam $\left(40^{\prime \prime}\right)$. Assuming a dust opacity of $\kappa_{\mathrm{m}}$ of $1.85 \mathrm{~cm}^{2} / \mathrm{g}$ at $850 \mu \mathrm{m}$ for grains with thick ice mantles and gas density $n(\mathrm{H})=10^{6} \mathrm{~cm}^{-3}$ (Ossenkopf \& Henning 1994), the effective $\mathrm{H}_{2}$ column density is (Launhardt 1996)

$N\left(\mathrm{H}_{2}\right)=\frac{6.2 \times 10^{16} S_{v}^{\text {beam }} \lambda^{3} \mathrm{e}^{\frac{1.44 \times 10^{4}}{\mathrm{~T}_{\mathrm{d}} \lambda}}}{\kappa_{\mathrm{m}}(\lambda) \theta^{2}} \frac{Z_{\odot}}{Z}$

where $S_{v}^{\text {beam }}$ is the flux density in Jy/beam, $\lambda$ the wavelength in $\mu \mathrm{m}, Z / Z_{\odot}$ is the metallicity relative to the solar metallicity (we assume $Z / Z_{\odot}=1$ ) and $\theta$ is the $F W H M$ of the $100 \mathrm{~m}$ beam in arcseconds. Here we assume that the dust temperature $T_{\mathrm{d}}$ is approximately equal to the gas temperature. We derive an effective $\mathrm{H}_{2}$ column density of the order of $6-20 \times 10^{22} \mathrm{~cm}^{-2}$ toward the peaks of the dust emission. Together with the $\mathrm{NH}_{3}$ column density, this is used to determine the ammonia abundance $\left(\chi_{\mathrm{NH}_{3}}=N\left(\mathrm{NH}_{3}\right) / N\left(\mathrm{H}_{2}\right)\right)$. We determine the peak effective $\mathrm{H}$ column density from the SCUBA map and the $\mathrm{NH}_{3}$ column density for the same position for each clump and get an upper limit for the fractional $\mathrm{NH}_{3}$ abundance as listed in Table 3. The average abundance is $\sim 4 \times 10^{-8}$, however has a 


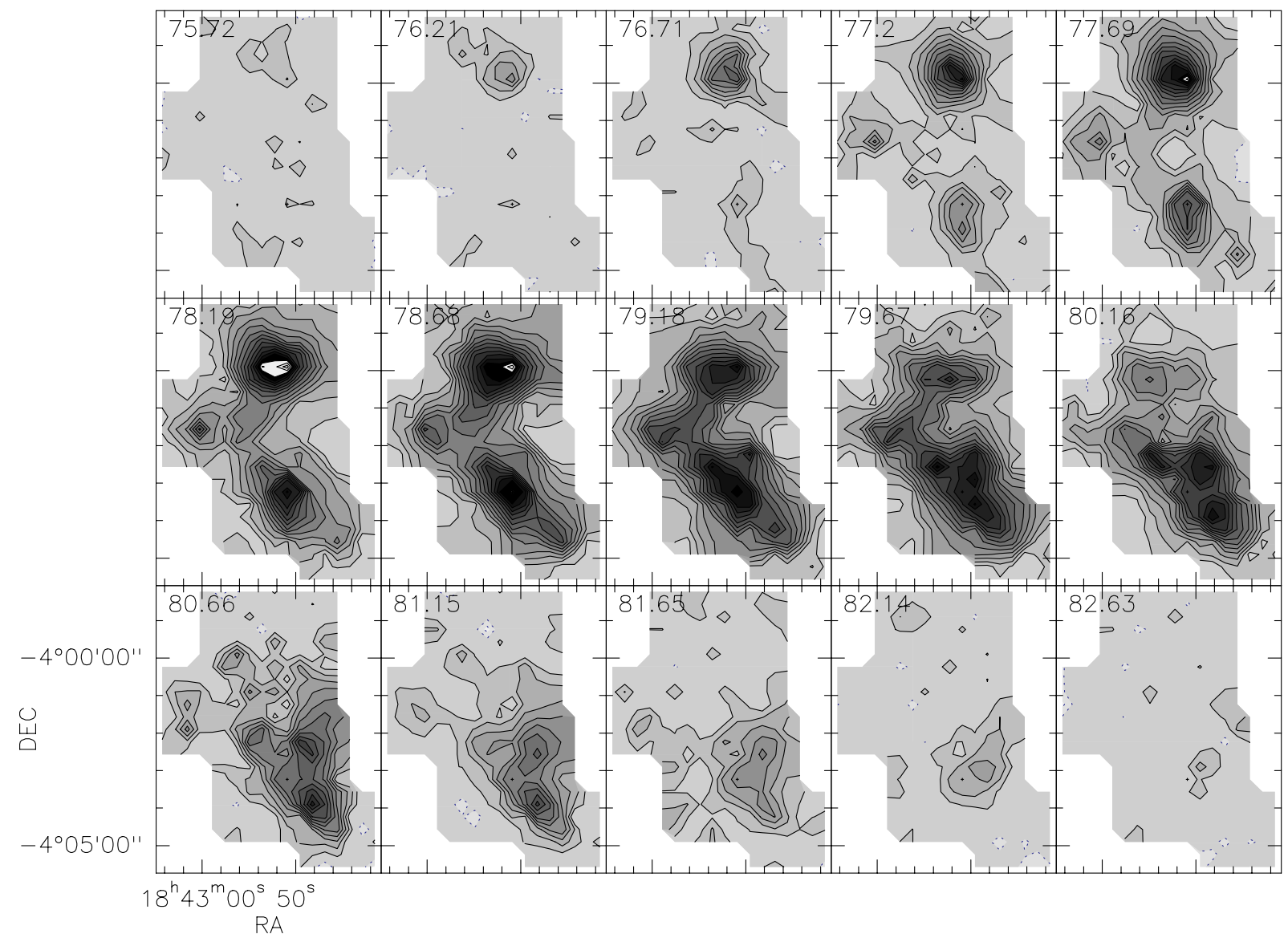

Fig. 8. channel map for $\mathrm{G} 28.34+0.06$ over the main component for the $\mathrm{NH}_{3}(1,1)$ transition.

low value towards $\mathrm{G} 79.27+0.38 \mathrm{NH}_{3} \mathrm{P} 2\left(7 \times 10^{-9}\right.$, as listed in Table 3).

The mass of the each cloud can be determined from the dust continuum and from $\mathrm{NH}_{3}$ emission. The gas mass is derived from the dust using the relation (Launhardt \& Henning 1997)

$M\left(\mathrm{H}_{2}\right)\left[M_{\odot}\right]=1.8 \times 10^{-14} \frac{S_{v} \lambda^{3} d^{2} \mathrm{e}^{\frac{1.44 \times 10^{4}}{\mathrm{~T}_{\mathrm{d}} \lambda}}}{\kappa_{m}(\lambda)} \frac{Z_{\odot}}{Z}\left[M_{\odot}\right]$

where $S_{v}$ is the integrated flux density in Jy and the other parameters are the same as in Eq. (5).

Our estimates for the mass and $\mathrm{H}_{2}$ column density are in general agreement with the previous work of Carey et al. (2000) for the submillimeter cores.

The total gas mass can be derived from the $\mathrm{NH}_{3}$ column density maps assuming a uniform fractional abundance of the molecule, for those sources without dust continuum data. The gas mass derived from SCUBA observations can be directly compared with the virial mass estimate. The virial parameter (Bertoldi \& McKee 1992) for a clump is defined as

$\alpha=\frac{5 \sigma^{2} R}{G M}$

where $\sigma$ is the three dimensional root mean square (rms) velocity dispersion and $R$ is the radius of the clump, and $M$ is the gas mass. Note that $\sigma=\sqrt{3 / 8 \ln 2} \times F W H M$. The virial mass is defined as $M_{\text {vir }}=5 \sigma^{2} R / G$. For the clumps to be stable against collapse, $\alpha \sim 1$. We find an average value of 2.1 for the virial parameter toward the individual cores. As the uncertainty in the dust opacity alone is a factor of $2, \alpha \sim 1$, and most of these cores appear to be virialised. Therefore, the core structure is consistent with them being supported by turbulent pressure without any evidence of external bounding pressures. G28.34+0.06 NH $\mathrm{NH}_{3} \mathrm{P} 4$ is an extreme case, where $\alpha=6.53$.

\section{Discussion}

\subsection{Gas temperature and distribution}

In G11.11-0.12, the position $\mathrm{NH}_{3} \mathrm{P} 2$ is a faint MSX source with no counterpart in the visible or NIR; it is very likely that this is a heavily embedded protostar (Pillai et al. 2005). The southern clumps are colder by $\sim 2-3 \mathrm{~K}$ than the northern clumps. Thus, these southern filaments may be at an earlier stage of evolution than the cores belonging to the northern filament, where star formation activity might heat up the gas. The core to core variation in temperature within an IRDC is observed toward several other sources (see Sect. 4.5). Therefore, these dense cores in IRDCs are ideal sites for investigating the initial conditions in forming a massive star.

The gas temperatures we derive generally agree with the dust temperatures of Carey et al. (2000) based on submm observations and an assumed dust emissivity index $\beta=1.75 . T_{\text {gas }}$ might be lower limits for those sources that show up as bright and compact objects in the submm dust emission, indicating 


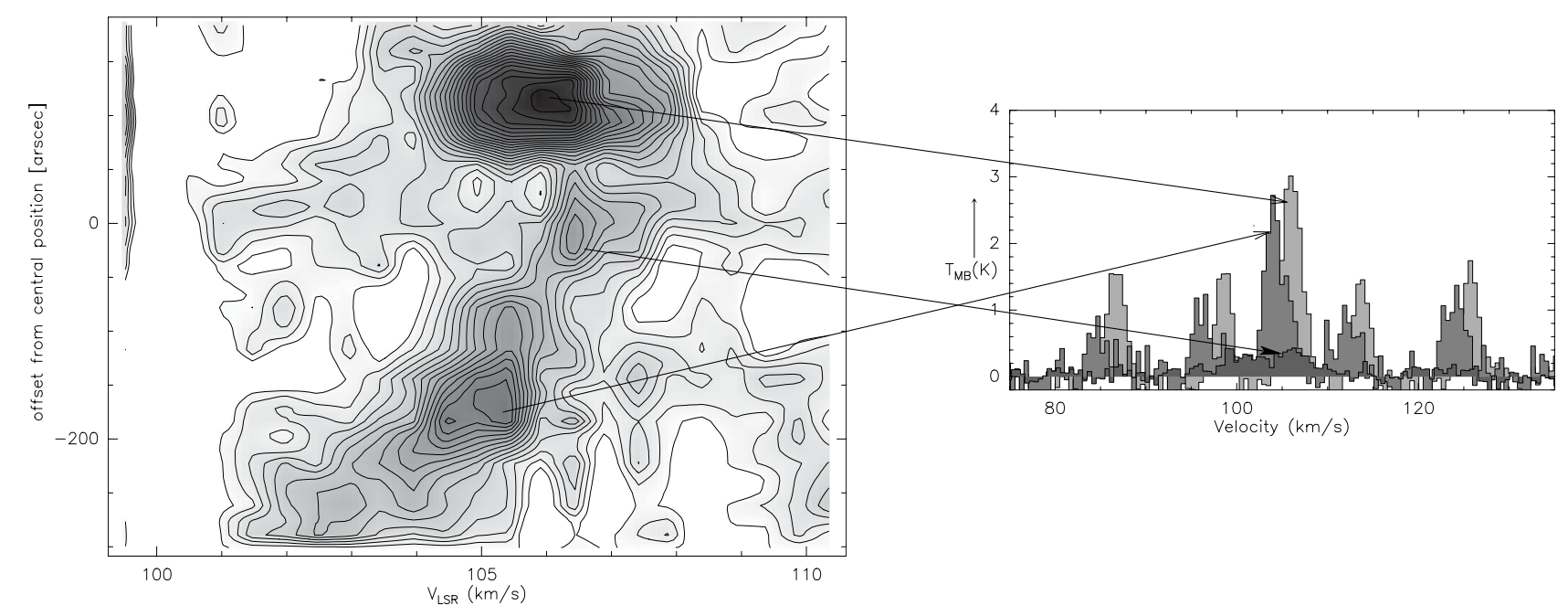

Fig. 9. Left panel: the average velocity-position map of $\mathrm{NH}_{3}(1,1)$ along the declination axis for the cloud G33.71-0.01. Right panel: the spectra obtained at the corresponding position in the map (indicated by the arrows). Note the large linewidth component toward the centre part of the cloud and the change in LSR velocity from south to north.

the presence of a heavily embedded object, but reasonable for the cold gas envelope.

\subsection{Physical properties, chemistry and lifetime}

The excellent correlation between the $\mathrm{NH}_{3}$ line and submm continuum emission is consistent with the predictions of chemical models that $\mathrm{NH}_{3}$ is relatively more abundant in high density region than other molecules (Bergin 2003). The column densities translate to extremely high extinction values of 55-450 mag. The discrepancies in $\mathrm{NH}_{3}$ and $\mathrm{H}_{2} \mathrm{CO}$ linewidths reported by Carey et al. (1998) (see Sect. 4.4) indicate the differences in the gas volume traced by the two molecules. The line wings seen in $\mathrm{H}_{2} \mathrm{CO}$ and $\mathrm{CH}_{3} \mathrm{OH}$ (Leurini et al. 2006, submitted) gas toward some sources are most likely high-velocity outflows. The critical density of the $\mathrm{H}_{2} \mathrm{CO}$ lines studied $\left(10^{6} \mathrm{~cm}^{-3}\right)$ is much higher than that of the $\mathrm{NH}_{3}$ inversion lines $\left(10^{4} \mathrm{~cm}^{-3}\right)$. Hence, while physical parameters derived from $\mathrm{NH}_{3}$ are representative of the general, cool IRDC material, $\mathrm{H}_{2} \mathrm{CO}$ probes the dense gas, which might be influenced by embedded protostars. The derived $\mathrm{NH}_{3}$ abundance of $0.7-10.1 \times 10^{-8}$ together with the centrally condensed $\mathrm{NH}_{3}$ emission is consistent with the chemical model predicted for pre-protostellar cores (Bergin \& Langer 1997). These values imply that $\mathrm{NH}_{3}$ is overabundant by factors of $5-10$ relative to "normal" (=lower density) and less turbulent dark clouds. In contrast, $\mathrm{H}_{2} \mathrm{CO}$ is under-abundant by a factor of $\sim 50$ (Carey et al. 1998). Hence the IRDCs appear to exhibit a complex chemistry.

We derive the flow crossing time, for the cloud to disperse due to its own internal motions. Perturbations within the cloud would disintegrate the cloud unless the propagation wavelength is much smaller than the distance across the cloud which it traverses. The estimated dynamical timescales of $\sim 0.5-2 \mathrm{Myrs}$, provide an upper limit to the life time of these clouds.

The virial masses for the different clumps span a wide range from 50 to $7880 M_{\odot}$. As typical for intermediate to high mass star-forming regions, most of the masses are skewed to values $>500 M_{\odot}$. The virial parameter $\sim 1$ for most of the clumps which are stable against collapse.

\subsection{IRDCs and other star-forming regions}

In a comparison of our $\mathrm{NH}_{3}$ results with the potential low mass counterpart B68 (Lai et al. 2003), we find that the linewidths and the virial masses are much higher towards our cores. The sizes and the column density and most importantly the masses we derive are also significantly higher, thus making IRDCs potential candidates as sites of high-mass star formation. The $(1,1)$ and $(2,2)$ linewidths for IRDCs are slightly lower but the $\mathrm{NH}_{3}$ column densities are comparable to the massive dense cloud identified in $\mathrm{NH}_{3}$ in $\mathrm{NGC} 6334 \mathrm{I}(\mathrm{N})$ by Kuiper et al. (1995). In terms of masses and sizes and temperatures, our sources are similar to those of Garay et al. (2004). Star formation probably has already started in some of them.

In Table 4, we present the mean properties of the dense cores of our sample with those of the cores in Taurus, Perseus and the Orion A complex as given in Ladd et al. (1994). It is evident that IRDC cores are on average highly turbulent, larger with much higher masses than the other cores while having similar temperature as the other cores. The IRDC masses derived here are for an $\mathrm{NH}_{3}$ abundance of $10^{-7}$ as used by Ladd et al. (1994). But this is almost an order of magnitude higher than what we derive and hence the mean mass quoted in Table 4 will be an order of magnitude higher, several $1000 M_{\odot}$ instead of several 100. Compared to local dark clouds, IRDCs pile up significantly large amounts of mass and have supersonic internal motions. But how much of this mass goes into forming stars of low, intermediate or high mass is yet to be answered.

Tan (2005) find that a group of local IRDCs have masses of a few $10^{3}-10^{4} M_{\odot}$ and mean surface density of $\Sigma \sim 0.1 \mathrm{gcm}^{-2}$. They find that this is 3 times the mean surface density of a Giant Molecular Cloud (GMC) and very similar to the values found in more evolved systems like the Orion Nebula Cluster 
(ONC). Subsequently, they suggest that IRDCs forming from GMCs are the initial conditions for star clusters.

Tan (2005) define $\Sigma$ as $\Sigma=M /\left(\pi R^{2}\right)$, and if we compute the mean surface density for our sample and the local dark clouds (the mean Mass and the size as given in Table 4), we arrive at values between $0.08-0.4$. If the mean surface density were to be a measure of star formation efficiency, then IRDCs have a higher value compared to Taurus but not very much higher than Perseus which is understood to be an intermediate star forming region.

Studies of relatively local cluster forming regions like the $\rho$ Ophiuchus cloud, the Serpens molecular cloud and Orion B molecular cloud (Motte et al. 1998; Testi \& Sargent 1998; Johnstone et al. 2001) find that the mass spectrum of their cores are very similar to that of the stellar IMF. If this is indeed true, then the fraction of the core mass going into forming stars would be independent of mass and the stellar IMF would mainly be determined by the cloud fragmentation process (Blitz \& Williams 1999). If we assume a star formation efficiency of $30 \%$ in an IRDC core of mean mass $\sim 500 M_{\odot}$ and adopt the standard IMF (with power law indices $2.3 \pm 0.3$, $1.3 \pm 0.5$ and $0.3 \pm 0.7$ for masses $>0.5,0.08-0.5$ and $<0.05 M_{\odot}$ respectively), then $\sim 116$ stars could form in the core. Out of this, $\sim 2$ stars could be of high-mass stars $\left(\geq 8 M_{\odot}\right)$ while 64 would be low-mass/intermediate stars $\left(0.5 \leq M / M_{\odot} \leq 8\right)$ while the rest would be very low mass and sub-stellar objects. Indeed there is growing evidence of star formation in these cores (Rathborne et al. 2005; Pillai et al. 2005; Ormel et al. 2005).

\subsection{IRDCs Formed by Interaction with SNRs?}

From the velocity structure, G11.11-0.12 $\mathrm{NH}_{3} \mathrm{P} 2$ appears blue shifted while the clump $\mathrm{NH}_{3} \mathrm{P} 1$ towards the north and $\mathrm{NH}_{3} \mathrm{P} 4$ towards the south appears redshifted relative to the LSR velocity. This is thus not a case of smooth velocity gradient along the filament. It might be possible to explain the observed velocity structure, if we assume that the entire filament seen in projection is part of a unbound system where P1 and P4 lie at the same distance along the line-of-sight (l.o.s) at diametrically opposite ends while P2 is further in the front. A massive winddriven process which might have taken place in the close vicinity of the cloud could explain such a structure. Recent wide field MIR images of this region released from Spitzer show that this cloud has a filamentary concave structure spread over several parsecs with a significant density enhancement in the center (Menten et al. 2005). The morphology bears remarkable similarity to the structures predicted by 3-D numerical calculations simulating the impact of a planar shock front on an isolated globule (Boss 1995). The best known case of a ongoing SN-cloud interaction is in IC 443, where a $10^{4} \mathrm{yr}$ old SNR in the GEM OB1 association with a shock speed of $40 \mathrm{~km} \mathrm{~s}^{-1} \mathrm{im}-$ pacts the cloud resulting in highly excited molecular gas with very broad linewidths. However, a weaker shock (likely from a more evolved SNR) would result in temporary distortion and compression of the molecular cloud, followed by rebound to a equilibrium. An evolved SNR at an age of $10^{5} \mathrm{yr}$ with a shock speed of $100 \mathrm{~km} \mathrm{~s}^{-1}$, is one of the three likely weak shock waves that Boss (1995) propose to influence a cloud without destroying it. Such a shock wave would have already traversed 25 pc. Brogan et al. (2004) recently discovered the supernova remnant G11.03-0.05 which is within $7^{\prime}$ of the cloud. They claim that the SNR is not young.

The SNR has a shell-like appearance with a diameter of $\sim 8^{\prime}$ and very weak emission at centimeter wavelengths. Brogan et al. (2004) find that a pulsar PSRJ1809-1917 is located about $8.5^{\prime}$ from the SNR and is probably associated with the SNR. Considering the respective uncertainties of their distance estimates, the pulsar $(4 \pm 1 \mathrm{kpc})$ and G11.11-0.12 (3.6 $\pm 0.5 \mathrm{kpc})$ appear at or near the same distance.

In G33.71-0.01, we find another where the cloud seems to have undergone a shock at the centre (see Fig. 9). Note that there is a large linewidth towards the central core of this filament. The $(0,0)$ velocity is also different from the cores toward the south and north segment of the filament. The SNR G33.6+0.1 is within $5^{\prime}$ of this core and previous observations by Green (1989) shows an unusually broad $\mathrm{OH}$ absorption feature near $105 \mathrm{~km} \mathrm{~s}^{-1}$ towards this SNR. The $\mathrm{HCO}^{+}$and ${ }^{12} \mathrm{CO} \mathrm{J}=1-0$ observations by Green \& Dewdney (1992) reveal material shocked by the interaction of the SNR with the adjacent molecular cloud. The $2 \sigma$ detection of a large linewidth feature $\Delta v \geq 8 \mathrm{~km} \mathrm{~s}^{-1}$ centered at $105 \mathrm{~km} \mathrm{~s}^{-1}$ in $\mathrm{NH}_{3}(1,1)$ and $(2,2)$ might also be a sign of interaction of the cloud with the SNR.

All IRDCs except those in the Cygnus- $X$ region have a SNR in their vicinity, although an association, which would require observations with shock tracers, cannot be verified yet. To estimate the chance occurance of an IRDC and SNR on the sky, we sample $\sim 17.6$ square degrees in which (Green 2002) and Brogan et al. (2004) have identified a total of 22 remnants. The likelihood of a chance positional occurance within $7^{\prime}$ of an IRDC is small $(\leq 0.05)$ given a SNR surface density of 1.25 remnants per square degree. It is very likely that the SNRs are physically associated with the IRDCs G11.11 and G33.71-0.01.

\subsection{IRDCs in the framework of an evolutionary sequence}

To obtain general statistics on IRDCs we compare in Fig. 10 the core gas properties of our sample of 9 sources with the cores presented in the Jijina et al. (1999) $\mathrm{NH}_{3}$ database. The database consists of 264 dense cores, with and without associations with young stellar objects. For the large linewidths of IRDCs $\left(\Delta v>0.8 \mathrm{~km} \mathrm{~s}^{-1}\right)$, there is a distinct trend for the IRDCs to be colder relative to the cores from the database. The $\mathrm{NH}_{3}$ column density is also higher for the IRDCs, surpassed only by a few high column densities from low mass cores observed with the high angular resolution of the VLA, which evidently sees more of the core interiors that are possibly associated with YSOs.

In Fig. 11 we present a similar comparison with a sample of UCHII regions from the Wood \& Churchwell (1989) catalogue and the sources from the Beuther et al. (2002) study of 

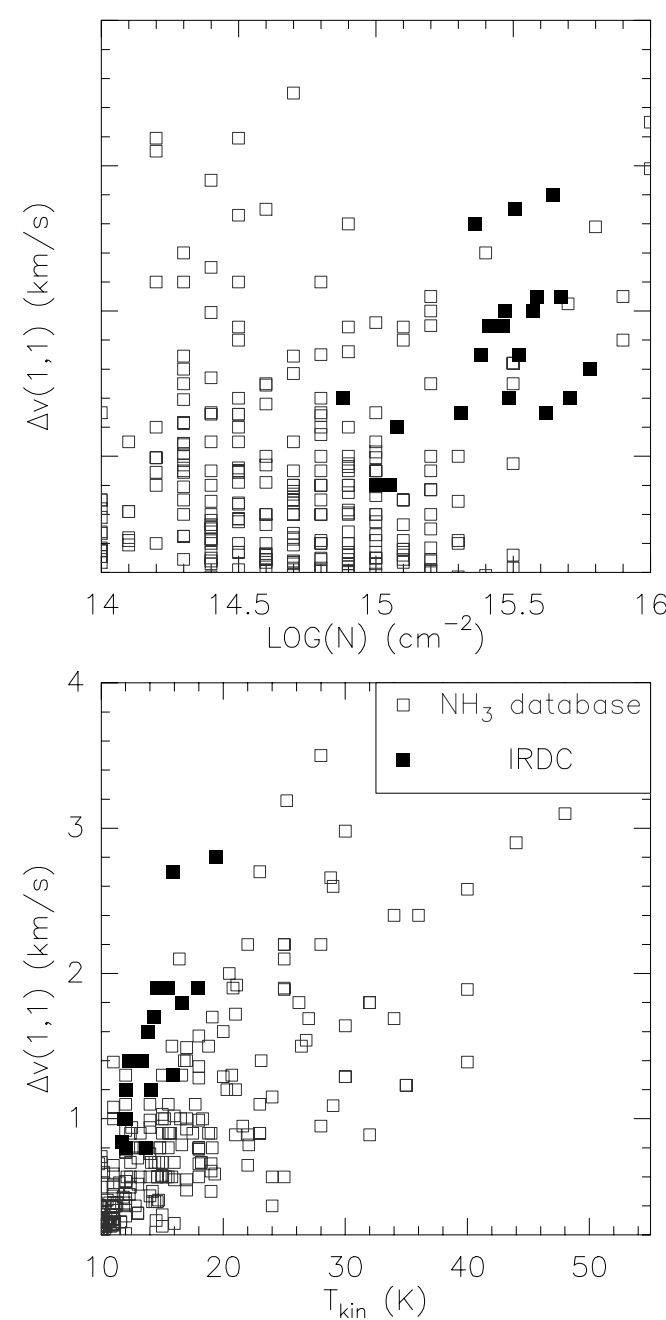

Fig. 10. Bottom panel: the correlation plot of kinetic temperature with the linewidths. The black filled squares indicate the IRDCs and the grey unfilled squares the cores from the $\mathrm{NH}_{3}$ database. IRDCs have larger velocity dispersions while the rotational temperatures are comparable. Top panel: the correlation plot of $\mathrm{NH}_{3}$ column density with the linewidths shows that the average column density of IRDCs are higher.

high-mass protostellar objects (HMPOs). While recent studies reveal that HMPOs are in a pre-UCHII region phase (Molinari et al. 2002; Beuther et al. 2002), the nature of the stage preceding HMPOs has not been studied in detail. IRDCs are ideal candidates for this pre-HMPO stage. In Fig. 11 we present properties of source samples believed to cover the earliest phases of massive star formation based on $\mathrm{NH}_{3}$ observations. There is no clear trend in linewidths, however IRDCs have a significantly lower average linewidth $\left(\Delta \bar{v}=1.51 \mathrm{~km} \mathrm{~s}^{-1}\right)$ than HMPOs $\left(\Delta \bar{v}=2.05 \mathrm{~km} \mathrm{~s}^{-1}\right)$ and UCHII regions $\left(\Delta \bar{v}=2.52 \mathrm{~km} \mathrm{~s}^{-1}\right)$. There is a distinct temperature trend from the low temperatures of the IRDCs $(\bar{T}=13.9 \mathrm{~K})$ to increasing temperatures for the IRAS selected high-mass objects $(\bar{T}=18.34 \mathrm{~K})$ and high temperatures for the objects associated with UCHII regions $(\bar{T}=22.6 \mathrm{~K})$. Temperature and linewidths must be understood as averages over the core and parts of the envelope since the beam is $40^{\prime \prime}$.
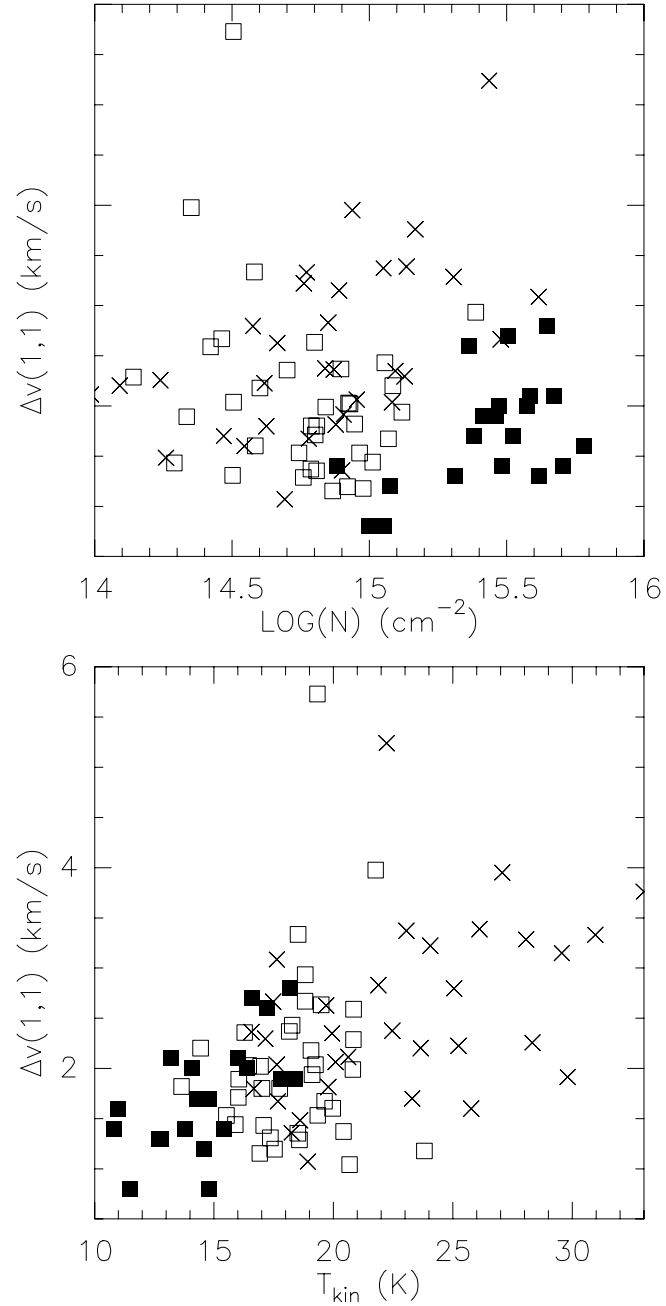

Fig. 11. Bottom panel: the correlation plot of kinetic temperature with the linewidth. The black filled squares indicate the IRDCs, the crosses the sources from the Wood and Churchwell catalogue and the unfilled squares Beuther et al. (2002) sources. IRDCs are colder and on average have lower linewidths. Top panel: the correlation plot of $\mathrm{NH}_{3}$ column density with the linewidths.

There is no clear trend in column density. However, on average the column densities of IRDCs are high compared to the other two samples. We interpret the clear trend in temperature and the tentative differences in $\mathrm{NH}_{3}$ linewidths and column densities $\left(N\left[\mathrm{NH}_{3}\right]\right)$ as possible manifestation of an evolutionary sequence. Starless cores on the verge of star formation are expected to be cold ( $T \leq 20 \mathrm{~K})$, to have high column densities and smaller linewidths. The temperature and the linewidth in a core will increase after the formation of an embedded protostar (via radiative heating and injection of turbulence through outflows and winds), while the envelope will be dispersed via outflows and winds and thus column densities will decrease with time.

One would expect the highest ammonia column densities for the hot sources due to evaporation of ammonia from the grains although in some cases IRDCs have higher column densities. However, this effect might be very localised to the hot cores $(0.1 \mathrm{pc}$ and smaller) and not be true for the larger scale emission. Additionally some molecular material might already 
Table 4. Mean values of $\mathrm{NH}_{3}$ core samples.

\begin{tabular}{rccccc}
\hline \hline Sample & $F W H M\left(\mathrm{~km} \mathrm{~s}^{-1}\right)$ & $T_{\text {kin }}(\mathrm{K})$ & size $(\mathrm{pc})$ & $M_{\mathrm{NH}_{3}}\left(M_{\odot}\right)$ & $\Sigma$ \\
\hline Taurus & 0.33 & 10 & 0.06 & 1.1 & 0.08 \\
Perseus & 0.55 & 13 & 0.12 & 9 & 0.16 \\
Orion & 1.12 & 17 & 0.15 & 21 & 0.24 \\
IRDC cores & 1.7 & 15 & 0.57 & 492 & 0.40 \\
\hline
\end{tabular}

Notes: Columns are the source sample, the mean $F W H M$ of the $\mathrm{NH}_{3}(1,1)$ line, kinetic temperature, size of the core, mass as determined from $\mathrm{NH}_{3}$ assuming a fractional abundance of $\mathrm{NH}_{3}$ relative to $\mathrm{H}_{2}$ and the mean surface density. The mean values for dense cores in Taurus, Perseus and Orion have been taken from Ladd et al. (1994).

be dispersed in the hot sources by the interaction of the young OB clusters with their environment.

\section{Conclusions}

In this paper, we discussed ammonia observations of a selected set of IRDCs and the derived physical properties. Our results are as summarised below.

The ammonia emission correlates very well with MIR absorption and ammonia peaks distinctly coincide with dust continuum peaks. Several cores are detected within the clouds with deconvolved sizes smaller than the $40^{\prime \prime} F W H M$ beam size. We can constrain the average gas temperature to between 10 and $20 \mathrm{~K}$.

We observe high linewidths $\left(1 \leq \Delta v / \mathrm{km} \mathrm{s}^{-1} \leq 3.5\right)$, hence turbulence plays an important role in the stability of an IRDC. There are significant velocity gradients observed between the cores. The effect of external shock/outflow tracers, on the gas kinematics is suggestive in some cases, but needs to be investigated further.

The column densities translate to extremely high $A_{\mathrm{V}}$ values (55-450 mag), therefore any active star formation would be heavily embedded. The total cloud gas mass derived from the $\mathrm{NH}_{3}$ data ranges from $10^{3}-10^{4} M_{\odot}$. The virial parameter is $\sim 1$ for most of the clumps, and the cores appear to be stable against gravitational collapse. As a result IRDCs are potential sites for star formation. If we were to adopt the stellar IMF and a star formation efficiency of $30 \%$, then every IRDC core could fragment to form $>100$ stars, with at least two high mass stars $\left(>8 M_{\odot}\right)$.

The fractional abundance of $\mathrm{NH}_{3}$ (relative to $\mathrm{H}_{2}$ ) is $0.7-10.0 \times 10^{-8}$. This, together with the excellent correlation in morphology of the dust and gas, is consistent with the time dependent chemical model for $\mathrm{NH}_{3}$ of Bergin \& Langer (1997) and implies that $\mathrm{NH}_{3}$ remains undepleted. The derived abundance is a factor 5-10 larger than that observed in local dark clouds while $\mathrm{H}_{2} \mathrm{CO}$ is underabundant by a factor of $\sim 50$. Hence, the chemistry governing these IRDCs might be complex and could be different from other parts of the dense ISM.

The time scales we derive for the clouds to disperse due to their own internal motions of a few Myrs provides an upper limit to the life time of these clouds. We suggest that SNRs might be the trigerring mechanism responsible for the formation of an IRDC.

The comparison of the physical properties from ammonia of our IRDCs sample with other source samples - HMPO's and
UCHIIs - strongly suggests that most of these IRDCs are the most likely candidates for pre-protostellar cores of massive star formation.

Acknowledgements. This research has made use of the NASA/ IPAC Infrared Science Archive, which is operated by the Jet Propulsion Laboratory, California Institute of Technology, under contract with the National Aeronautics and Space Administration. S.J.Carey acknowledges support from a NASA Long Term Space Astrophysics grant. T.Pillai was supported for this research through a stipend from the International Max Planck Research School (IMPRS) for Radio and Infrared Astronomy at the University of Bonn. T.Pillai thanks her colleague J.Kauffmann for useful discussions and comments on the manuscript.

\section{References}

Bachiller, R., Guilloteau, S., \& Kahane, C. 1987, A\&A, 173, 324

Bergin, E. A. 2003, in SFChem 2002: Chemistry as a Diagnostic of Star Formation, Proc. of a conference held August 21-23, 2002 at University of Waterloo, Waterloo, Ontario, Canada N2L 3G1, ed. C. L. Curry, \& M. Fich (Ottawa Canada: NRC Press), 63

Bergin, E. A., \& Langer, W. D. 1997, ApJ, 486, 316

Bertoldi, F., \& McKee, C. F. 1992, ApJ, 395, 140

Beuther, H., Schilke, P., Menten, K. M., et al. 2002, ApJ, 566, 945

Blitz, L., \& Williams, J. P. 1999, in The Origin of Stars and Planetary Systems, NATO ASIC Proc., 540, 3

Boss, A. P. 1995, ApJ, 439, 224

Brogan, C. L., Devine, K. E., Lazio, T. J., et al. 2004, AJ, 127, 355

Carey, S. J., Clark, F. O., Egan, M. P., et al. 1998, ApJ, 508, 721

Carey, S. J., Feldman, P. A., Redman, R. O., et al. 2000, ApJ, 543, L157

Egan, M. P., Shipman, R. F., Price, S. D., et al. 1998, ApJ, 494, L199

Evans, N. J., Shirley, Y. L., Mueller, K. E., \& Knez, C. 2002, in ASP Conf. Ser. 267: Hot Star Workshop III: The Earliest Phases of Massive Star Birth, 17

Forveille, T., Guilloteau, S., \& Lucas, R. 1989, Grenoble: IRAM

Garay, G., Faúndez, S., Mardones, D., et al. 2004, ApJ, 610, 313

Green, D. A. 1989, MNRAS, 238, 737

Green, D. A. 2002, VizieR Online Data Catalog, 7227, 0

Green, D. A., \& Dewdney, P. E. 1992, MNRAS, 254, 686

Hennebelle, P., Pérault, M., Teyssier, D., \& Ganesh, S. 2001, A\&A, 365,598

Ho, P. T. P., \& Townes, C. H. 1983, ARA\&A, 21, 239

Jijina, J., Myers, P. C., \& Adams, F. C. 1999, ApJS, 125, 161

Johnstone, D., Fich, M., Mitchell, G. F., \& Moriarty-Schieven, G. 2001, ApJ, 559, 307

Johnstone, D., Fiege, J. D., Redman, R. O., Feldman, P. A., \& Carey, S. J. 2003, ApJ, 588, L37 
Kuiper, T. B. H., Peters, W. L., Forster, J. R., Gardner, F. F., \& Whiteoak, J. B. 1995, ApJ, 446, 692

Ladd, E. F., Myers, P. C., \& Goodman, A. A. 1994, ApJ, 433, 117 Lai, S.-P., Velusamy, T., Langer, W. D., \& Kuiper, T. B. H. 2003, AJ, 126,311

Launhardt, R. 1996, Ph.D. Thesis

Launhardt, R., \& Henning, T. 1997, A\&A, 326, 329

Menten, K. M., Pillai, T., \& Wyrowski, F. 2005, in IAU Symp., 23

Molinari, S., Testi, L., Rodríguez, L. F., \& Zhang, Q. 2002, ApJ, 570, 758

Motte, F., Andre, P., \& Neri, R. 1998, A\&A, 336, 150

Ormel, C. W., Shipman, R. F., Ossenkopf, V., \& Helmich, F. P. 2005, A\&A, 439, 613

Ossenkopf, V., \& Henning, T. 1994, A\&A, 291, 943
Ott, M., Witzel, A., Quirrenbach, A., et al. 1994, A\&A, 284, 331

Perault, M., Omont, A., Simon, G., et al. 1996, A\&A, 315, L165

Pillai, T., Wyrowski, F., Menten, K. M., \& Krügel, E. 2005, ArXiv Astrophysics e-prints

Rathborne, J. M., Jackson, J. M., Chambers, E. T., et al. 2005, ArXiv Astrophysics e-prints

Redman, R. O., Feldman, P. A., Wyrowski, F., et al. 2003, ApJ, 586, 1127

Tafalla, M., Myers, P. C., Caselli, P., \& Walmsley, C. M. 2004, A\&A, 416, 191

Tan, J. C. 2005, ArXiv Astrophysics e-prints

Testi, L., \& Sargent, A. I. 1998, ApJ, 508, L91

Teyssier, D., Hennebelle, P., \& Pérault, M. 2002, A\&A, 382, 624

Wood, D. O. S., \& Churchwell, E. 1989, ApJS, 69, 831 